\title{
Frequency Tuning of Piezoelectric Energy Harvesters Thanks to a Short-Circuit Synchronous Electric Charge Extraction
}

\author{
A. Morel ${ }^{1,2}$, G. Pillonnet ${ }^{1}$, P. Gasnier ${ }^{1}$, E. Lefeuvre ${ }^{3}$, A. Badel $^{2}$ \\ ${ }^{1}$ Univ. Grenoble Alpes, CEA, LETI, MINATEC, F-38000 Grenoble, France \\ ${ }^{2}$ Univ. Savoie Mont-Blanc, SYMME, F-74000, Annecy, France \\ ${ }^{3}$ Univ. Paris Sud - CNRS, Université Paris-Saclay, Centre for Nanoscience and Nanotechnology, F-91400, \\ Paris, France \\ Contact authors: adrien.morel@cea.fr, adrien.badel@univ-smb.fr
}

\begin{abstract}
This paper introduces a new electrical strategy for maximizing the energy scavenged from highly coupled and lowly damped piezoelectric energy harvesters. The proposed strategy named Short-Circuit Synchronous Electric Charge Extraction (SC-SECE) introduces a shortcircuit phase whose start time $\phi_{s}$ and duration $\Delta \phi$ can be adjusted. The control of $\phi_{s}$ and $\Delta \phi$ allows to electrically adapt the dynamics of the electromechanical harvester. In a first part, we analytically derive the influences of $\phi_{s}$ and $\Delta \phi$ on the harvester dynamics, and prove that they both affect the damping induced by the electrical interface on the mechanical resonator and its resonant frequency. Thereafter, we numerically evaluate the SC-SECE performances as a function of the intrinsic harvester's characteristics. We expose that if the coupling and/or mechanical quality factor of the harvester are important enough, the SC-SECE leads to enhanced power frequency responses compared to SECE and SEH strategies. Experimental results on a high coupling piezoelectric energy harvester associated with the SC-SECE strategy have been realized and are in good agreement with the analytical predictions. Under various vibrations amplitudes, the harvester's resonant frequency has been tuned on a frequency range as large as $35 \%$ of its natural resonant frequency. We have been able to harvest more than $92 \mu \mathrm{W}$ for vibration frequencies ranging from $90 \mathrm{~Hz}$ to $140 \mathrm{~Hz}$, under an external acceleration of $0.2 G$.
\end{abstract}

Keywords - Energy harvesting, Nonlinear electrical interface, Piezoelectricity, Damping control, Frequency tuning, Synchronous Electric Charge Extraction.

\section{Introduction}

Wireless sensor nodes designed to facilitate monitoring, risk prevention, and more generally improve comfort in our day-to-day life, are increasingly present in our everyday environment. Most of these devices require a fairly small amount of energy to sense, process, then send an information. This makes energy harvesting an attractive alternative to electrochemical batteries, as it could lead to devices combining compactness, long lifetime, and robustness to harsh environments [1]. In closed and confined environments, where the thermal gradients and solar radiations are relatively weak, the major energy source can be found under the form of mechanical vibrations [2]. This may be the case in biomedical applications, for instance, to power implantable medical devices which are placed inside the human body. Here, compactness as well as autonomy are undeniably vital [3].

Among electromechanical transducers, piezoelectric materials are of interest because of their good trade-off between power density and technological maturity [4]. In order to amplify the mechanical strain applied on such materials, they are usually deposited on a linear mechanical oscillator having a relatively high quality factor. The harvested power from such electromechanical system is optimal when the damping induced by the energy extraction is equal to the mechanical intrinsic damping in the structure, and when the ambient vibration frequency matches the resonant 
frequency of the mechanical oscillator [5]. However, the harvested power drastically decreases when a slight mismatch appears between those two frequencies. This mismatch may be caused by many reasons: inaccurate design of the harvester leading to a difference between the expected and real resonant frequencies, aging of the mechanical oscillator, change in the ambient vibration spectrum, among others [6].

In order to face this challenge, researchers have proposed mechanical solutions based on nonlinear oscillators to enlarge the harvesting frequency bandwidth. The nonlinearities are usually induced thanks to nonlinear springs [7], buckled beam configurations [8], or magnetic forces [9], leading, in the most common cases, to softening, hardening, and bistable behaviors [10]. Nonetheless, these solutions are not flawless, as the hysteretic frequency response of such oscillators may lead to low displacement orbits, hindering the benefits of this approach [11]. Furthermore, the shapes of those harvesters' power frequency responses are highly dependent on the vibrations' amplitude.

Recently, some researchers proposed to adapt the dynamics of the piezoelectric energy harvester using the electrical interface connected to the piezoelectric material. Indeed, thanks to the indirect piezoelectric effect, the voltage across the piezoelectric material induces a force on the mechanical oscillator. Depending on its phase and amplitude, this force has an influence on both the harvester resonant frequency and damping [12]. The phase and amplitude of the piezoelectric force depending on the equivalent resistive and reactive components of the electrical load connected to the piezoelectric material, this phenomenon can also be understood as an electromechanical impedance matching, as explained extensively in [13].

Based on these principles, W.-J. Wu et al. first proposed in 2006 to adapt a linear capacitive load connected to the harvester, which allows to electrically tune the stiffness of the harvester, and impacts its electromechanical resonant frequency [14]. Further studies of the capacitive compensation have been proposed later, in 2009 [15] and 2013 [16]. A. Badel et al. proposed in 2014 a nonlinear interface called the Frequency Tuning Synchronous Electric Charge Extraction (FTSECE), which consisted in adapting the amount of electric charges collected every semi-period of vibration, and the phase of the harvesting events [17]. Y. Cai et al. proposed in parallel a similar approach, analyzing this strategy using equivalent impedances methods [18]. In 2015, P. H. Hsieh et al. proposed to add a tunable switching delay to the Parallel Synchronized Switch Harvesting on Inductance (P-SSHI) [19]. Thereafter, B. Zhao et al. proposed to combine the idea of adding a tunable switching delay with the synchronized multiple bias-flip (SMBF) concept [20], which consists in optimizing the charges inversion processes thanks to multiple voltage-changing steps [21]. In 2017, our group developed a novel approach to electrically tune the resonant frequency of piezoelectric energy scavengers and enlarge their harvesting bandwidth: the N-SECE, which consists in harvesting the energy at a different frequency than the vibration's frequency [22]. Recently, we proposed another concept, the SC-SECE, which relies on a tunable short-circuit phase [23].

In this paper, we propose a thorough and extended analysis of the SC-SECE, and we compare it with traditional electrical interfaces (Full bridge rectifier, SECE), and other strategies to enlarge the bandwidth (i.e. using nonlinear mechanical oscillators and other tunable electrical interfaces). SC-SECE allows to adapt both the resonant frequency and the damping of a linear Piezoelectric Energy Harvester (PEH), thanks to a short-circuit phase whose start angular time $\phi_{S}$ and angular duration $\Delta \phi$ are both adjustable. First, we propose a model of the voltage and displacement waveforms associated with the SC-SECE, and derive an analytical expression of the harvested power, depending on the harvesting frequency, the harvester characteristics, and the two electrical parameters $\phi_{s}$ and $\Delta \phi$. Thereafter, we numerically analyze our model in order to determine the power frequency responses of PEH associated with the electrical interface implementing the SC-SECE strategy. In a last part, we experimentally verify the validity of our analytical predictions, using a highly coupled 
piezoelectric harvester connected to an electrical interface implementing the SC-SECE strategy. The experimental results are then commented, compared to the theoretical ones, and the proposed SCSECE strategy is finally compared to other wideband solutions.

\section{Electromechanical system model}

\subsection{Piezoelectric harvester modelling}

A linear piezoelectric energy harvester is usually made of a piezoelectric material deposited upon a mechanical resonator. Around its first resonant frequency, such a system can be modeled by a single mechanical degree of freedom system, as depicted in Fig.1 [24].

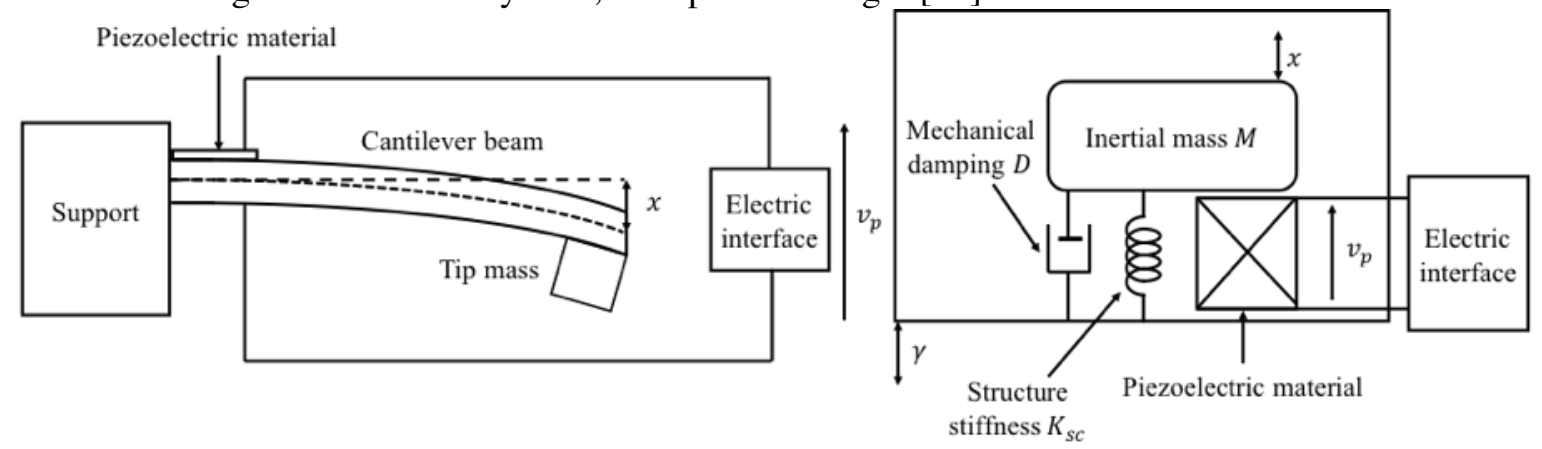

Figure 1 - Linear piezoelectric harvester made of a cantilever beam and its equivalent single degree of freedom modelling

The mechanical resonator can be, for instance, a cantilever beam of stiffness $K_{s c}$ whose free end has been fixed to an inertial mass $M$, and subject to frictional forces modelled by a linear damping coefficient $D$. Due to the indirect piezoelectric effect, the piezoelectric material exerts a force on the mechanical resonator, which is, in first approximation, proportional to the voltage across the piezoelectric element, $v_{p}$. Under an external acceleration $\gamma$, the second order differential equation governing the displacement $x$ of the inertial mass is then given by (1).

$$
-M \gamma=M \ddot{x}+D \dot{x}+K_{s c} x+\alpha v_{p}
$$

Where $\alpha$ is the force factor, and is dependent on both the mechanical resonator's geometry and the beam and piezoelectric material's properties. The piezoelectric material, under the strain induced by the resonator oscillation, generates electrical charges. These charges are either stored in the material dielectric capacitance $C_{p}$, or harvested by the electrical interface. The differential equation governing the electrical dynamics of the harvester is given by (2).

$$
\alpha \dot{\mathrm{x}}=\mathrm{C}_{\mathrm{p}} \dot{\mathrm{v}_{\mathrm{p}}}+\mathrm{i}_{\mathrm{p}}
$$

Where $i_{p}$ accounts for the current drawn by the electrical interface. The electromechanical model introduced by (1) and (2) is accurate as long as the mechanical linearity hypothesis is true, the dielectric losses in the piezoelectric material are small and the influences of higher mechanical modes remain negligible. Under a harmonic excitation, and as we assume that the mechanical oscillator has a 
relatively important quality factor, we assume that the inertial mass displacement is only a sinusoidal signal of magnitude $X_{m}$, as expressed by (3).

$$
\mathrm{x}=\mathrm{X}_{\mathrm{m}} \cos (\omega \mathrm{t})=\mathrm{X}_{\mathrm{m}} \cos (\theta)
$$

Where $\omega$ is the angular frequency of the harmonic excitation. In order to facilitate analytical developments, several dimensionless variables have been introduced in previous works [12], and are summarized in (4).

$$
\left\{\begin{array}{c}
\omega_{0}=\sqrt{\mathrm{K}_{\mathrm{sc}} / \mathrm{M}} \\
\Omega_{\mathrm{m}}=\omega / \omega_{0} \\
\mathrm{k}_{\mathrm{m}}^{2}=\alpha^{2} /\left(\mathrm{K}_{\mathrm{sc}} \mathrm{C}_{\mathrm{p}}\right) \\
\mathrm{Q}_{\mathrm{m}}=\sqrt{\mathrm{K}_{\mathrm{sc}} \mathrm{M} / \mathrm{D}}
\end{array}\right.
$$

Where $\omega_{0}$ is the short-circuit angular resonant frequency of the mechanical resonator, and $\Omega_{m}$ is the normalized ambient vibration frequency, $k_{m}^{2}$ is the modified coupling factor of the harvester, and corresponds to the ratio of the maximum electrostatic energy stored in the piezoelectric material divided by the maximum elastic energy stored in the mechanical resonator under quasi static excitation. $Q_{m}$ accounts for the quality factor of the mechanical resonator. The maximum harvestable power expression with a linear PEH excited under a harmonic acceleration has been analytically determined in previous works $[12,25]$ and is given by (5). This limit can only be reached when the harvester is working at resonance, and when the damping induced by the electrical interface is equal to the mechanical damping.

$$
P_{\max }=\frac{|M \gamma|^{2}}{8 D}=\frac{|\gamma|^{2} Q_{m} M}{8 \omega_{0}}
$$

\subsection{Standard SECE strategy}

E. Lefeuvre et al. first proposed the SECE strategy in [26] as an alternative to Standard Energy Harvesting (SEH) interfaces, as it allows to harvest more energy for lowly coupled / highly damped piezoelectric harvesters, when the product $k_{m}^{2} Q_{m}<\pi / 4$. Furthermore, contrarily to SEH and Synchronized Switch Harvesting on Inductance (SSHI) interfaces, its efficiency does not depend on the voltage across the storage element, and hence does not require a Maximum Power Point Tracking (MPPT) loop working constantly to optimize the harvested power [27, 28]. In the standard SECE strategy, the interface circuit is not connected to the piezoelectric element during most of the vibration's period. Most of the time, the piezoelectric harvester works in open-circuit configuration, and all the charges generated, thanks to the piezoelectric effect, are stored in the dielectric capacitance $C_{p}$. When the voltage reaches an extremum, an inductance is briefly connected to the piezoelectric material, forming a LC resonator. All the electrostatic energy stored in the piezoelectric material is then quickly transferred in the inductance. When the voltage becomes $0 \mathrm{~V}$, meaning that all the energy has been transferred from the piezoelectric material to the electrical circuit, the inductance is disconnected from the piezoelectric material, the energy stored in the inductance is transferred in a storage capacitor and the harvester restarts to operate in open-circuit configuration. The energy harvested during a single harvesting event can be calculated using (6). 


$$
\mathrm{W}_{\mathrm{SECE}}=\frac{1}{2} \mathrm{C}_{\mathrm{p}} \mathrm{v}_{\mathrm{m}}^{2}
$$

Where $v_{m}$ stands for the piezoelectric voltage extremum value. The piezoelectric voltage and inertial masse displacement waveforms, as well as a typical interface circuit implementing the SECE strategy are shown in Fig. 2 and Fig. 3. If the SECE strategy is efficient when associated with lowly coupled / highly damped PEHs, it has however been proven that for highly coupled / lowly damped piezoelectric harvesters $\left(k_{m}^{2} Q_{m}>\pi / 4\right)$, the SECE strategy draws too much power from the mechanical resonator [29]. This overdamps the mechanical resonator, leading to a reduced inertial mass displacement amplitude, and low harvested energy performances. Furthermore, the energy harvested with the SECE interface remains fairly limited when the piezoelectric harvester is excited outside the resonance.

\subsection{SC-SECE strategy}

The proposed SC-SECE strategy's aim is to overcome the standard SECE weaknesses thanks to the introduction of a tunable short-circuit phase. This short-circuit phase allows to limit the amount of energy converted from the mechanical resonator to the piezoelectric material, hence avoiding any overdamping effect when $k_{m}^{2} Q_{m}>\pi / 4$ [22]. First, let's define $\phi_{s} \in[0, \pi]$ as the angle at which the short-circuit phase starts. In order not to waste any energy, $\phi_{s}$ is associated as well with the energy harvesting events, which does not necessarily happen on the voltage extrema anymore (corresponding to $\left.\phi_{s}=0\right)$. We define as well $\Delta \phi \in[0, \pi]$ as the angular time spent in short-circuit condition. The piezoelectric voltage and inertial mass displacement waveforms are shown in Fig. 2, and a systemlevel implementation of the SC-SECE is shown in Fig. 3. Compared to the standard SECE system's implementation [26], the SC-SECE only requires an additional switch and a few control blocks. These additional requirements do not cost much space as they can all easily be integrated. The choice of the electrical parameters $\left(\phi_{S}, \Delta \phi\right)$ can either be done manually or could be automated thanks to an ultralow-power algorithm such as a gradient algorithm.

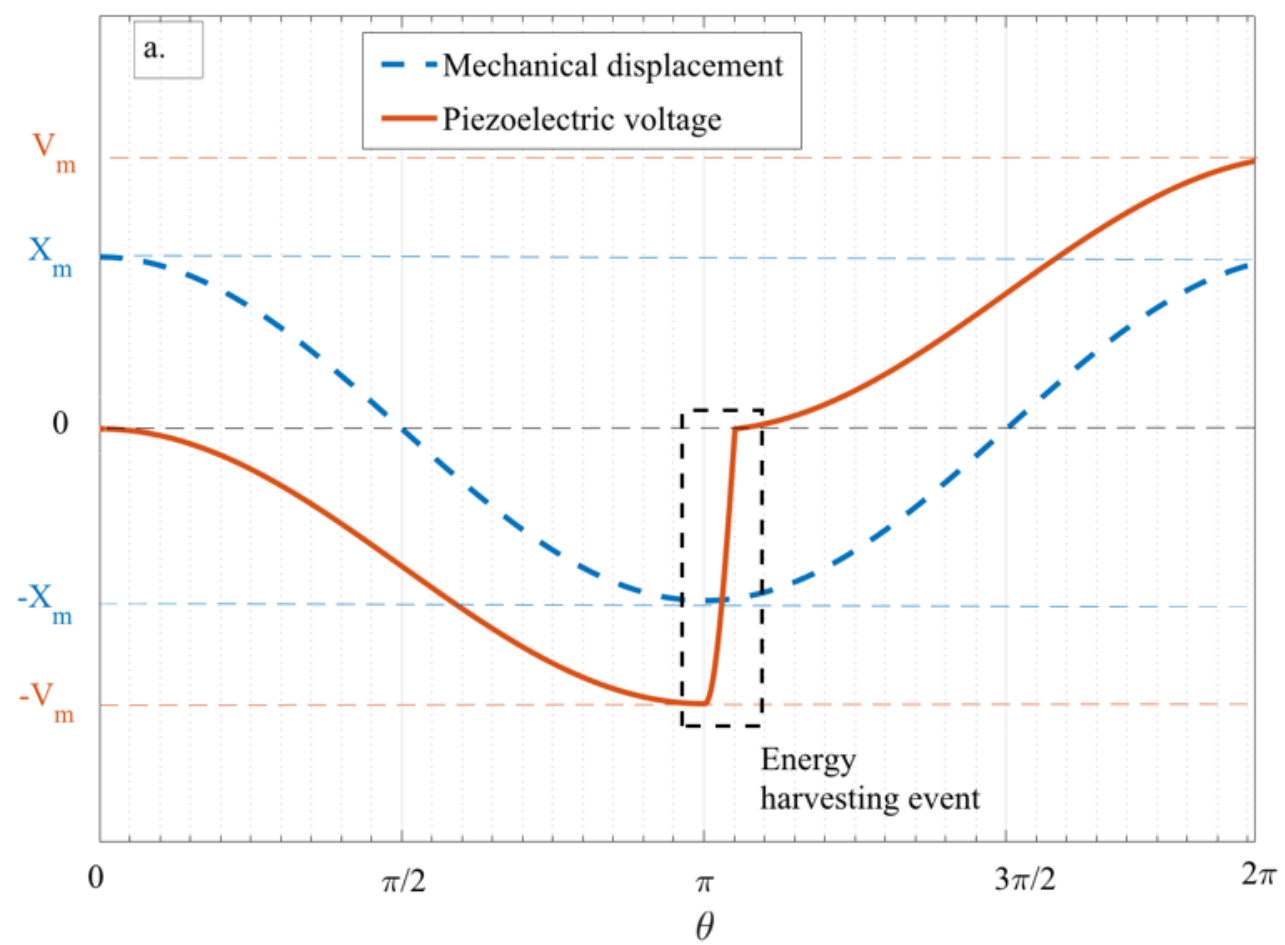




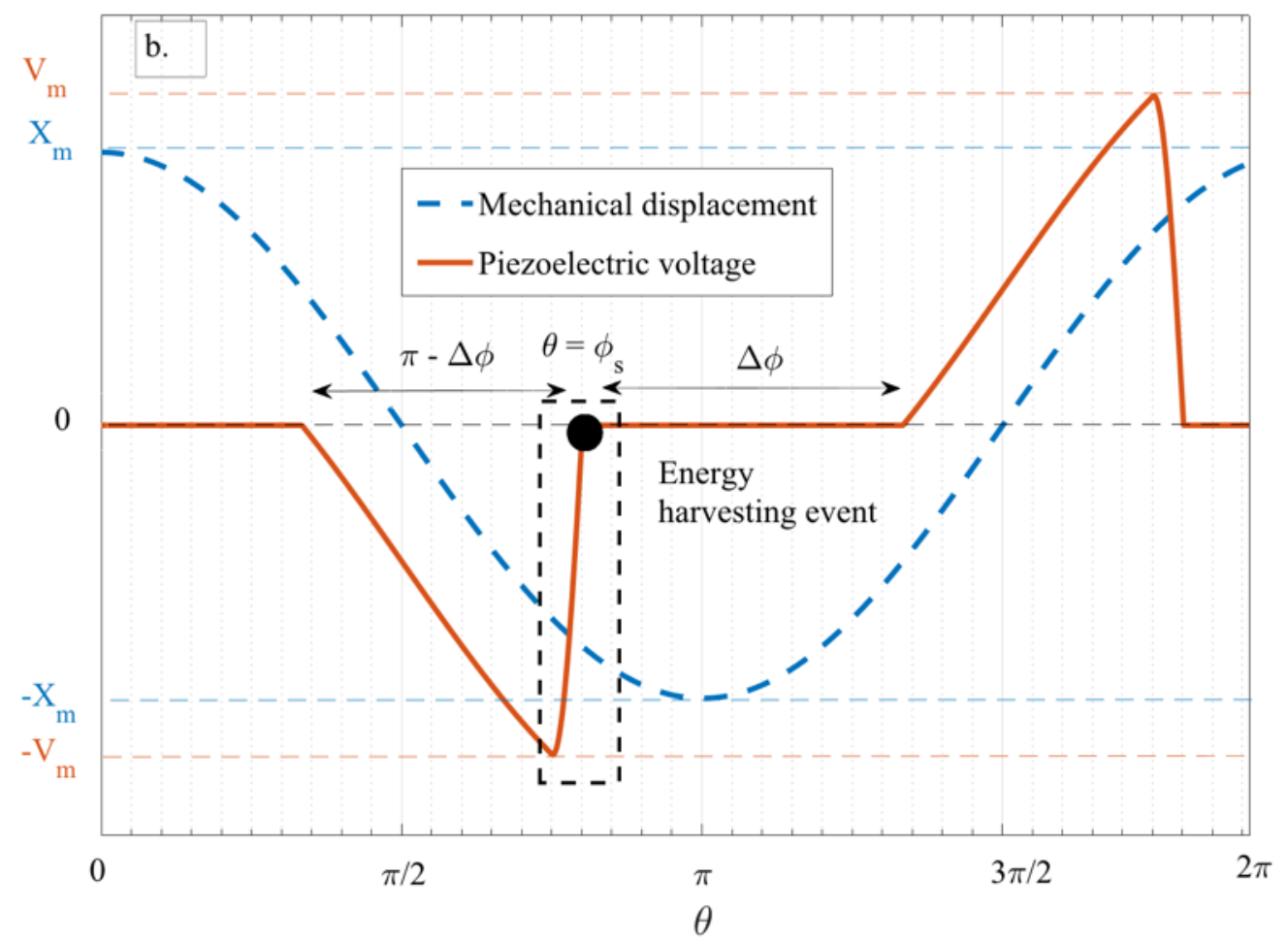

Figure 2 - Voltage and mechanical displacement waveforms associated with a. the standard SECE and b. the proposed SC-SECE

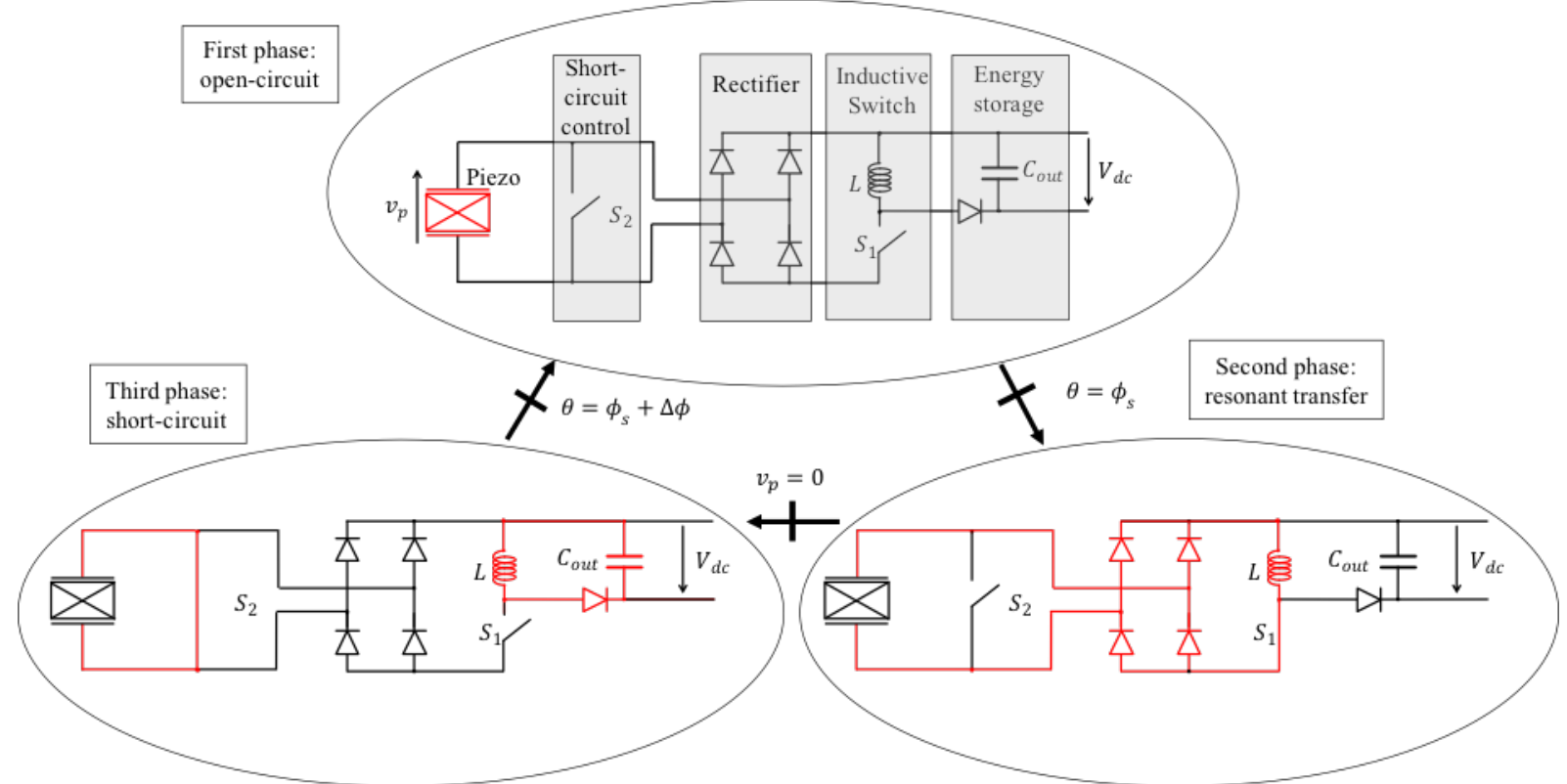

Figure 3 - Example of a system-level implementation of the proposed SC-SECE and its three different working phases.

From (2), the analytical expression of the piezoelectric voltage $v_{p}$ during a vibration's period $\left[\phi_{s}, \phi_{s}+2 \pi\right)$ can be expressed as a piecewise function of $x$, as shown in (7). 


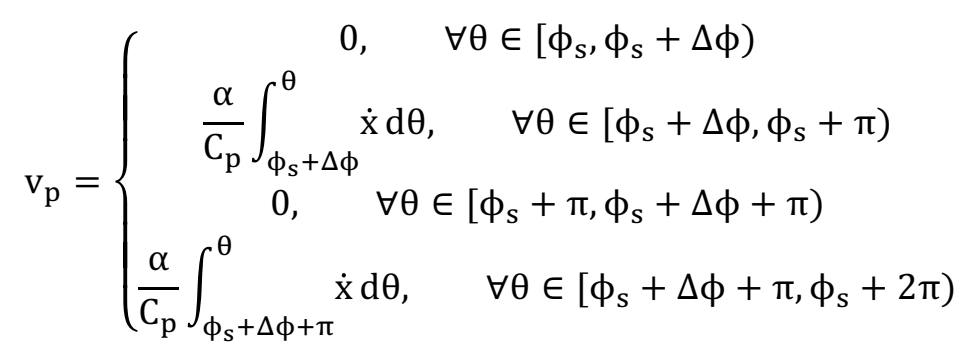

Since $v_{p}$ is a periodic function, we can find its associated Fourier series, expressed by (8).

$$
\mathrm{v}_{\mathrm{p}}=\mathrm{a}_{0}+\sum_{\mathrm{n}=1}^{\infty} \mathrm{a}_{\mathrm{n}} \cos (\mathrm{n} \theta)+\sum_{\mathrm{n}=1}^{\infty} \mathrm{b}_{\mathrm{n}} \sin (\mathrm{n} \theta)
$$

Where $a_{0}, a_{n}, b_{n}$ are the piezoelectric voltage series coefficients. Due to the filtering effect of the linear mechanical resonator, we assume that only the piezoelectric voltage fundamental component has an impact on the harvester dynamics. This assumption greatly simplifies the analytical calculations of the piezoelectric voltage. The fundamental $\left.v_{p}\right|_{1}$ of the piezoelectric voltage $v_{p}$ can be expressed as follows:

$$
\left.\mathrm{v}_{\mathrm{p}}\right|_{1}=\mathrm{a}_{1} \cos (\theta)+\mathrm{b}_{1} \sin (\theta)
$$

Applying Fourier series theory on (7), $a_{1}$ and $b_{1}$ can be found by solving (10).

$$
\left\{\begin{array}{l}
\mathrm{a}_{1}=\frac{2 \alpha}{\pi \mathrm{C}_{\mathrm{p}}} \int_{\phi_{\mathrm{s}}+\Delta \phi}^{\phi_{\mathrm{s}}+\pi}\left[\int_{\phi_{\mathrm{s}}+\Delta \phi}^{\theta} \dot{\mathrm{x}} \mathrm{d} \theta\right] \cos (\theta) \mathrm{d} \theta \\
\mathrm{b}_{1}=\frac{2 \alpha}{\pi \mathrm{C}_{\mathrm{p}}} \int_{\phi_{\mathrm{s}}+\Delta \phi}^{\phi_{\mathrm{s}}+\pi}\left[\int_{\phi_{\mathrm{s}}+\Delta \phi}^{\theta} \dot{\mathrm{x}} \mathrm{d} \theta\right] \sin (\theta) \mathrm{d} \theta
\end{array}\right.
$$

Injecting (3) in (10) leads to the following Fourier coefficient expressions:

$$
\left\{\begin{array}{c}
\mathrm{a}_{1}=\frac{\alpha \mathrm{X}_{\mathrm{m}}}{\pi \mathrm{C}_{\mathrm{p}}}\left[\pi-\Delta \phi+\frac{\sin \left(2 \phi_{\mathrm{s}}+2 \Delta \phi\right)}{2}+\frac{\sin \left(2 \phi_{\mathrm{s}}\right)}{2}+2 \cos \left(\phi_{\mathrm{s}}+\Delta \phi\right) \sin \left(\phi_{\mathrm{s}}\right)\right] \\
\mathrm{b}_{1}=-\frac{\alpha \mathrm{X}_{\mathrm{m}}}{\pi \mathrm{C}_{\mathrm{p}}}\left[\cos \left(\phi_{\mathrm{s}}\right)+\cos \left(\phi_{\mathrm{s}}+\Delta \phi\right)\right]^{2}
\end{array}\right.
$$

Thus, combining (9) and (11) leads to the expression of the fundamental $\left.v_{p}\right|_{1}$ of the piezoelectric voltage in the frequency domain:

$$
\left\{\begin{array}{c}
\left.\underline{\mathrm{v}_{\mathrm{p}}}\right|_{1}=\frac{\alpha \underline{\mathrm{x}}}{\mathrm{C}_{\mathrm{p}}}\left[\varepsilon_{\mathrm{K}}+\mathrm{j} \varepsilon_{\mathrm{D}}\right] \\
\varepsilon_{\mathrm{K}}=1-\frac{\Delta \phi}{\pi}+\frac{\sin \left(2 \phi_{\mathrm{s}}+2 \Delta \phi\right)}{2 \pi}+\frac{\sin \left(2 \phi_{\mathrm{s}}\right)}{2 \pi}+\frac{2 \cos \left(\phi_{\mathrm{s}}+\Delta \phi\right) \sin \left(\phi_{\mathrm{s}}\right)}{\pi} \\
\varepsilon_{\mathrm{D}}=\frac{\left[\cos \left(\phi_{\mathrm{s}}\right)+\cos \left(\phi_{\mathrm{s}}+\Delta \phi\right)\right]^{2}}{\pi}
\end{array}\right.
$$

Where $\underline{x}, \varepsilon_{K}, \varepsilon_{D}$ are the displacement written in the frequency domain, and the normalized damping and stiffness induced by the electrical interface, respectively. Combining (1) and (12) in the 
frequency domain, and isolating $\underline{x}$ leads to the inertial mass displacement expression. Normalizing the obtained expression using the dimensionless variables introduced by (4), we get (13).

$$
\underline{x}=\frac{\omega_{0}^{2} \gamma}{\left(1-\Omega_{m}^{2}+k_{m}^{2} \varepsilon_{K}\right)+j\left(\Omega_{m} / Q_{m}+k_{m}^{2} \varepsilon_{D}\right)}
$$

Thus, the magnitude of the inertial mass displacement is expressed by (14).

$$
X_{m}=\frac{\left|\omega_{0}^{2} \gamma\right|}{\sqrt{\left(1-\Omega_{m}^{2}+k_{m}^{2} \varepsilon_{K}\right)^{2}+\left(\Omega_{m} / Q_{m}+k_{m}^{2} \varepsilon_{D}\right)^{2}}}
$$

The harvested energy, traditionally given by expression (6) for the standard SECE, becomes (15) in the case of the SC-SECE strategy.

$$
\mathrm{W}_{\mathrm{SC}-\mathrm{SECE}}=\frac{1}{2} \mathrm{C}_{\mathrm{p}} \mathrm{v}_{\mathrm{p}}\left(\phi_{\mathrm{s}}\right)^{2}=\frac{\alpha^{2} \mathrm{X}_{\mathrm{m}}^{2}}{2 \mathrm{C}_{\mathrm{p}}}\left[\cos \left(\phi_{\mathrm{s}}\right)+\cos \left(\phi_{\mathrm{s}}+\Delta \phi\right)\right]^{2}
$$

The power harvested thanks to the SC-SECE can thus be derived:

$$
\mathrm{P}_{\mathrm{SC}-\mathrm{SECE}}=\frac{\omega}{\pi} \mathrm{W}_{\mathrm{SC}-\mathrm{SECE}}=\frac{\omega \alpha^{2} \mathrm{X}_{\mathrm{m}}^{2}}{2 \pi \mathrm{C}_{\mathrm{p}}}\left[\cos \left(\phi_{\mathrm{s}}\right)+\cos \left(\phi_{\mathrm{s}}+\Delta \phi\right)\right]^{2}
$$

Combining (14) and (16), we can get the following power expression:

$$
\mathrm{P}_{\mathrm{SC}-\mathrm{SECE}}=\frac{\mathrm{k}_{\mathrm{m}}^{2}|\mathrm{M} \gamma|^{2} \omega\left[\cos \left(\phi_{\mathrm{s}}\right)+\cos \left(\phi_{\mathrm{s}}+\Delta \phi\right)\right]^{2}}{2 \pi \mathrm{K}_{\mathrm{sc}}\left[\left(1-\Omega_{\mathrm{m}}^{2}+\mathrm{k}_{\mathrm{m}}^{2} \varepsilon_{\mathrm{K}}\right)^{2}+\left(\Omega_{\mathrm{m}} / \mathrm{Q}_{\mathrm{m}}+\mathrm{k}_{\mathrm{m}}^{2} \varepsilon_{\mathrm{D}}\right)^{2}\right]}
$$

This power expression can be normalized with respect to the maximum harvestable power, whose expression is given by (5). The normalized power $P_{\text {norm }} \in[0,1]$ is given by (18). $P_{\text {norm }}$ gives an insight about how efficient the mechanical-to-electrical conversion is.

$$
\mathrm{P}_{\text {norm }}=\frac{\mathrm{P}_{\mathrm{SC}-\mathrm{SECE}}}{\mathrm{P}_{\max }}=\frac{4 \mathrm{k}_{\mathrm{m}}^{2} \Omega_{\mathrm{m}}\left[\cos \left(\phi_{\mathrm{s}}\right)+\cos \left(\phi_{\mathrm{s}}+\Delta \phi\right)\right]^{2}}{\mathrm{Q}_{\mathrm{m}} \pi\left[\left(1-\Omega_{\mathrm{m}}^{2}+\mathrm{k}_{\mathrm{m}}^{2} \varepsilon_{\mathrm{K}}\right)^{2}+\left(\Omega_{\mathrm{m}} / \mathrm{Q}_{\mathrm{m}}+\mathrm{k}_{\mathrm{m}}^{2} \varepsilon_{\mathrm{D}}\right)^{2}\right]}
$$

\section{Numerical analysis of the tuning parameters influences}

Based on the previously derived analytical expression of the harvested power (18), we compute the power frequency responses of the proposed SC-SECE strategy. For every vibration's frequency, the parameters $\left(\phi_{s}, \Delta \phi\right)$ are optimized in order to maximize the harvested power. Figures 4, 5 and 6 present the SECE (dashed lines), SEH (dots), and SC-SECE (straight lines) power frequency responses for PEH exhibiting a low coupling $\left(k_{m}^{2}=0.01\right)$, medium coupling $\left(k_{m}^{2}=0.08\right)$, and high coupling $\left(k_{m}^{2}=0.4\right)$ respectively, for a given $Q_{m}=20$. 


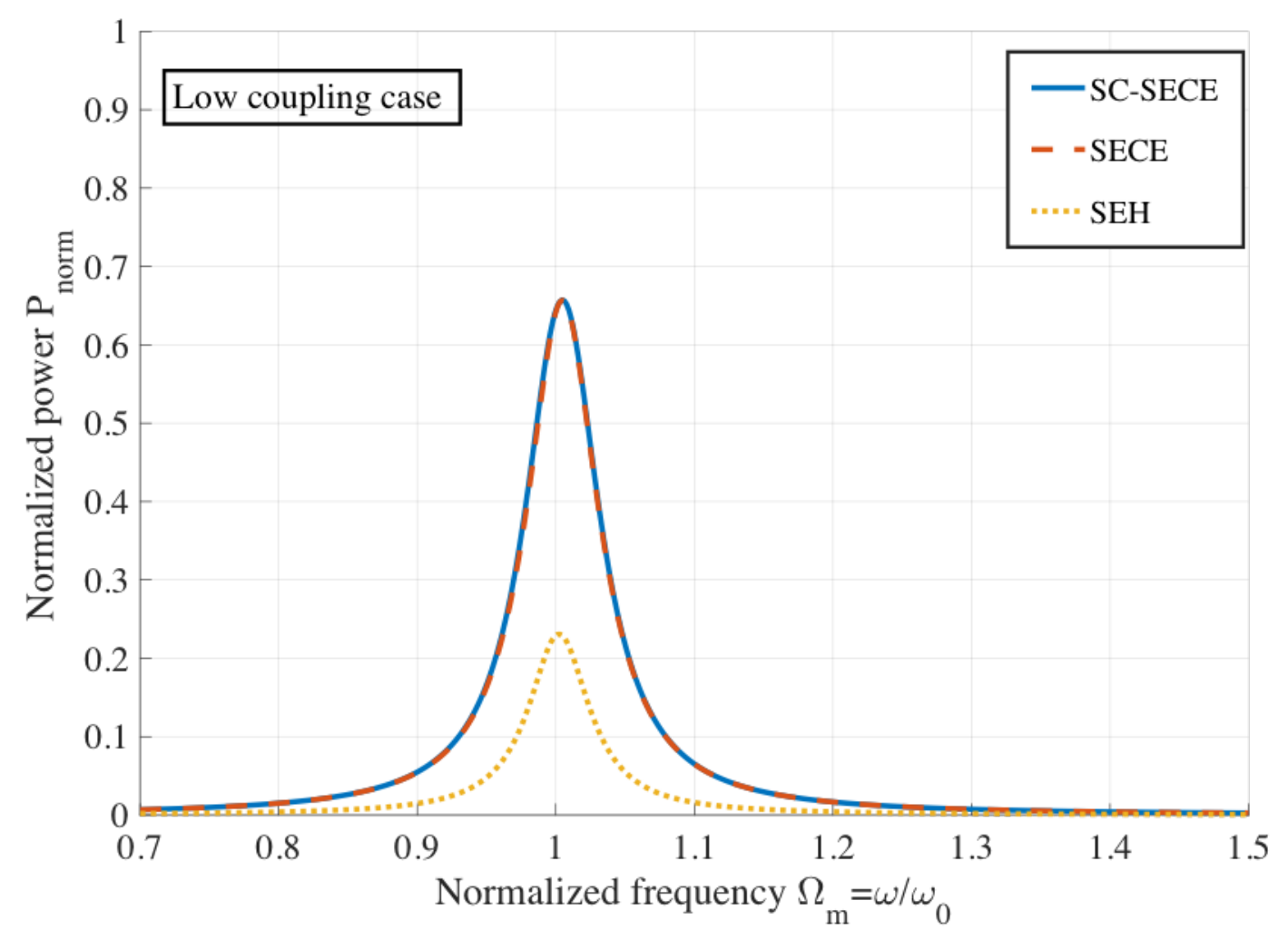

Figure 4 - Power frequency responses of a lowly coupled PEH, with $Q_{m}=20$ and $k_{m}^{2}=0.01$

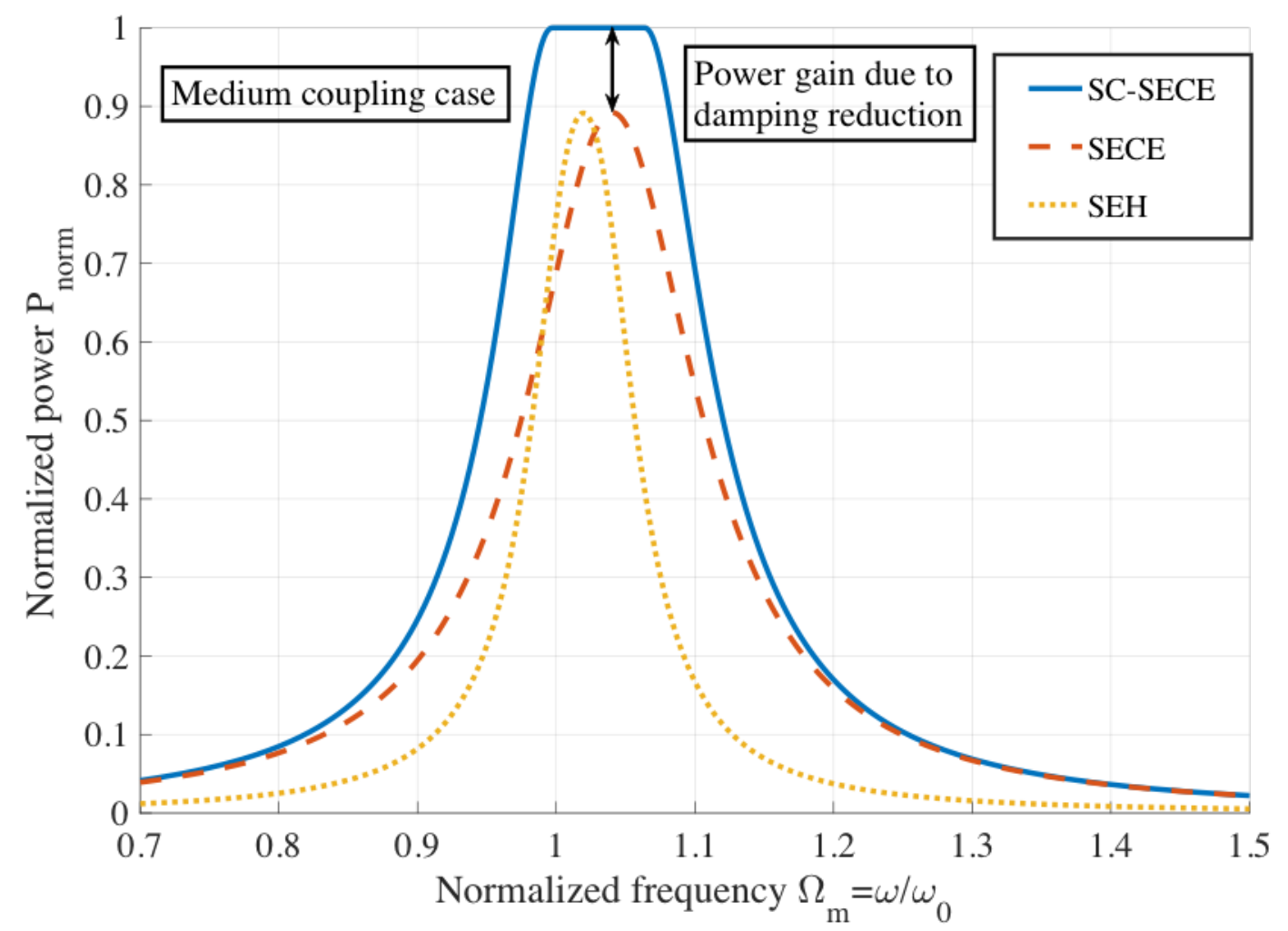

Figure 5 - Power frequency responses of a moderately coupled PEH, with $Q_{m}=20$ and $k_{m}^{2}=0.08$ 


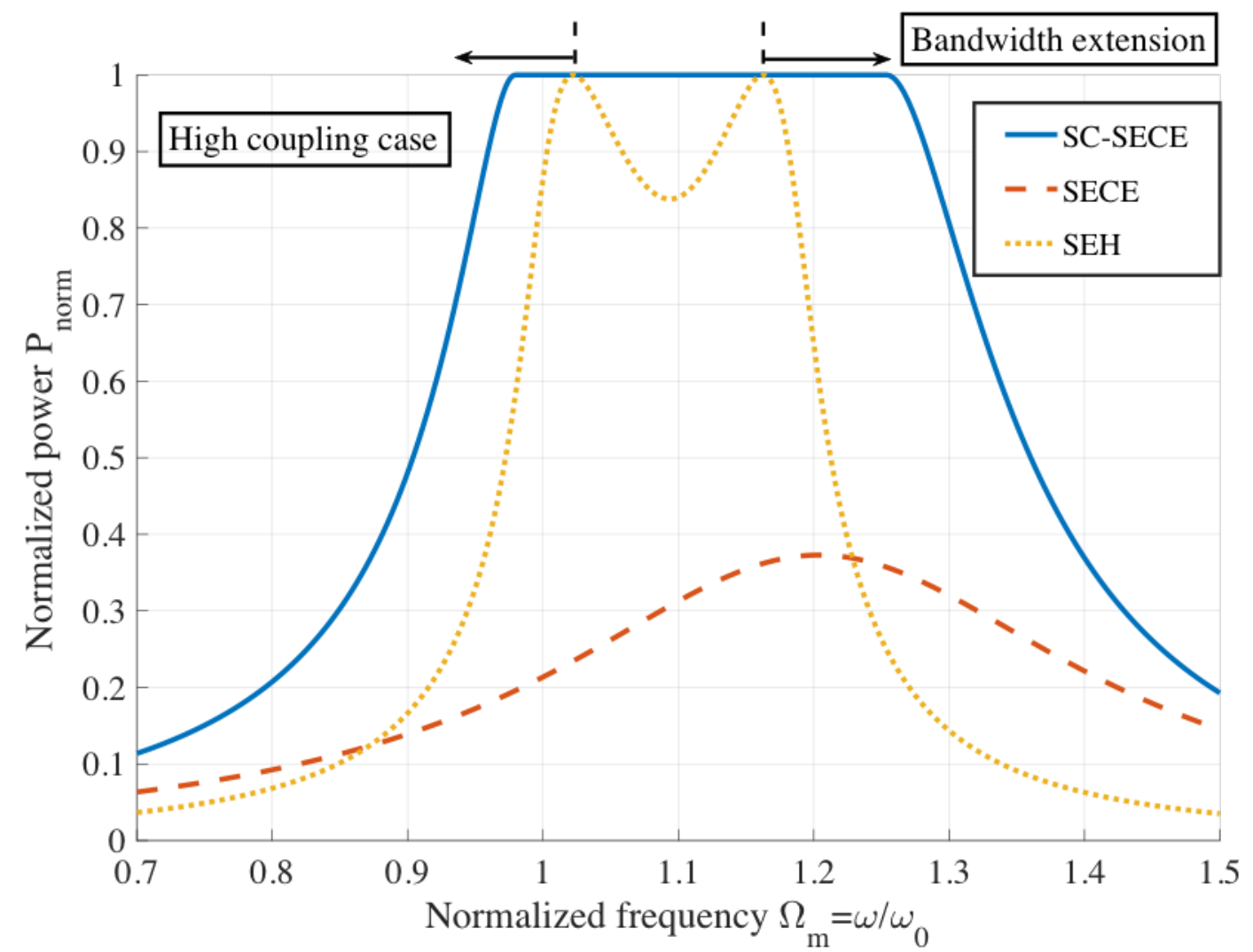

Figure 6 - Power frequency responses of a highly coupled PEH, with $Q_{m}=20$ and $k_{m}^{2}=0.4$

For low coupling harvesters, the power frequency responses of the SECE and SC-SECE strategies are almost superimposed. Thus, in this case, there is little to no interest to include a shortcircuit after the harvesting event. As predicted, both the SECE and SC-SECE strategies are more efficient than the SEH. As the coupling increases, it can be observed that the maximum power of the standard SECE strategy decreases, which is due to the overdamping phenomena extensively explained in $[22,29]$. In another hand, the SC-SECE maximum power remains equal to the maximum harvestable power $P_{\max }$. Furthermore, the bandwidth of the SC-SECE becomes more and more important as the coupling is increased. 


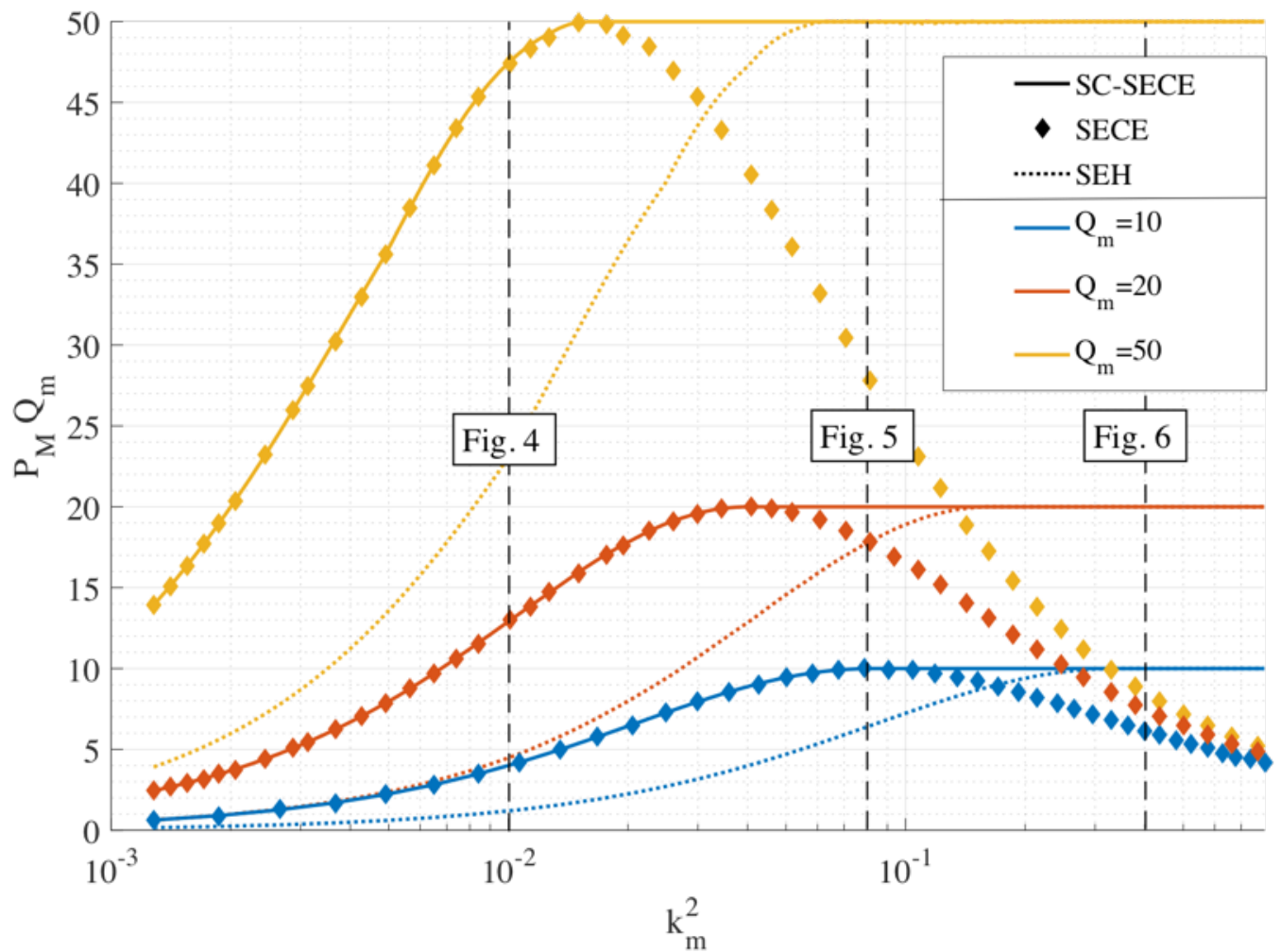

Figure $7-P_{M} Q_{m}$ as a function of $k_{m}^{2}$ and for $Q_{m}=10$ in blue, $Q_{m}=20 \mathrm{in} \mathrm{red,} \mathrm{and} Q_{m}=50 \mathrm{in}$ yellow.

The product of the maximum normalized power $P_{M}=\max \left(P_{\text {norm }}\right)$ times $Q_{m}$ is a good indicator of how much power can be harvested from a PEH associated with a particular electrical strategy [12]. Indeed, $P_{M}$ gives an insight of the mechanical-to-electrical conversion efficiency, while the net maximum power that can be harvested is directly proportional to the quality factor of the mechanical resonator $Q_{m}$, as shown by (5). This product has been computed in Fig.7 as a function of $k_{m}^{2}$, and for various mechanical quality factor $Q_{m}$. Here as well, we can observe that for lowly coupled / highly damped piezoelectric harvesters (when the product $k_{m}^{2} Q_{m}$ is small), both the SECE and SCSECE allow to harvest more power than the SEH strategy. When the coupling gets higher, the maximum harvested power with the SECE interface starts decreasing, while both the SC-SECE and the SEH strategy allow to harvest the maximum harvestable power $\left(P_{\text {norm }}=1\right)$. This confirms the trends that appears in Fig.4, Fig.5 and Fig. 6. For the highly coupled PEH introduced in next section $\left(Q_{m}=20, k_{m}^{2}=0.4\right)$, the SC-SECE allows to harvest up to 2.6 times more energy than with the SECE strategy. 


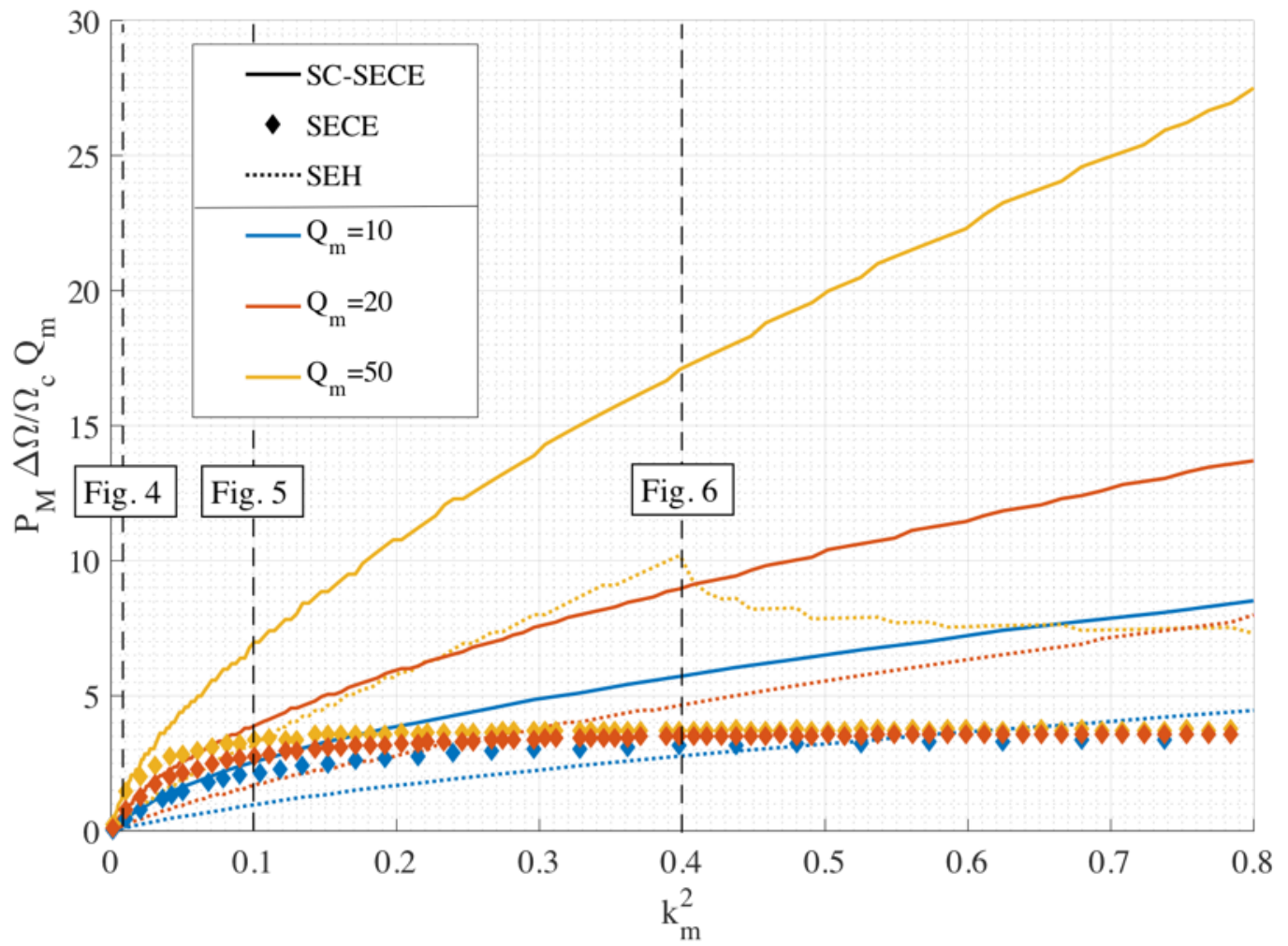

Figure 8 - Product $P_{M} Q_{m} \Delta \Omega / \Omega_{C}$ as a function of $k_{m}^{2}$ and for $Q_{m}=10$ in blue, $Q_{m}=20$ in red, and $Q_{m}=50$ in yellow.

Fig.8 shows the product of the normalized maximum harvested power $P_{M}$ with the quality factor of the mechanical resonator $Q_{m}$ and with the normalized harvesting bandwidth $\Delta \Omega / \Omega_{C}$, where $\Delta \Omega$ corresponds to the normalized frequency range where the harvested power is greater than half of the maximum harvested power, and $\Omega_{C}$ the central frequency of this frequency range. This product is computed as a function of the squared coupling coefficient $k_{m}^{2}$ and with various mechanical quality factors $Q_{m}$. We can observe that $P_{M} Q_{m} \Delta \Omega / \Omega_{C}$ is generally increasing with $k_{m}^{2}$. We can however notice that for high $k_{m}^{2}$ values, this product stops increasing for the SECE interface, because of the overdamping effect. For high values of $Q_{m}$ and $k_{m}^{2}$, this product may even start decreasing in the case of SEH. In another hand, in the case of the SC-SECE, this product gets always greater with a higher $k_{m}^{2}$, and is always more important than with any other strategy. This confirms the potential of the SCSECE to both optimize the harvested power $P_{M}$ from lowly and highly coupled PEH, while drastically increasing the power harvesting bandwidth $\Delta \Omega / \Omega_{C}$.

\section{Experimental validation}

\subsection{Experimental setup}

Experimentations have been conducted in order to validate the proposed SC-SECE strategy. The experimental setup shown in Fig. 9 consists of an electromagnetic shaker, which converts the electrical signal delivered by the DSpace real-time control and acquisition board and amplified by a power amplifier into an acceleration. The input voltage delivered by the DSpace board is regulated in order to maintain a constant shaker's acceleration amplitude, thanks to an internal control loop. A highly coupled piezoelectric harvester made with $10 \times 5 \times 0.5 \mathrm{~mm}^{3}$ plates of PZN-PT single crystals by 
Microfine Company (Singapore) poled along the [011] axis and exhibiting a very high transverse coupling coefficient $k_{31}$ of 0.92 along the [100] axis has been designed, and is depicted in Figure 10. This harvester has been fixed on the electromagnetic shaker, and its electrodes are connected to an electrical circuit made of discrete components which allows to obtain the voltage waveforms of the proposed SC-SECE strategy. Here, the circuit only allows to extract energy, without storing it, the stored energy optimization as well as the interface topology choice being beyond the scope of this paper. The electrical signals controlling the two tunable parameters $\left(\phi_{s}, \Delta \phi\right)$ are sent from the DSpace board, and the voltage across the piezoelectric harvesters, as well as the positions of the harvester and its inertial mass are recorded through the DSpace board and stored on a computer for each parameter couple combination, and for each vibration frequency.

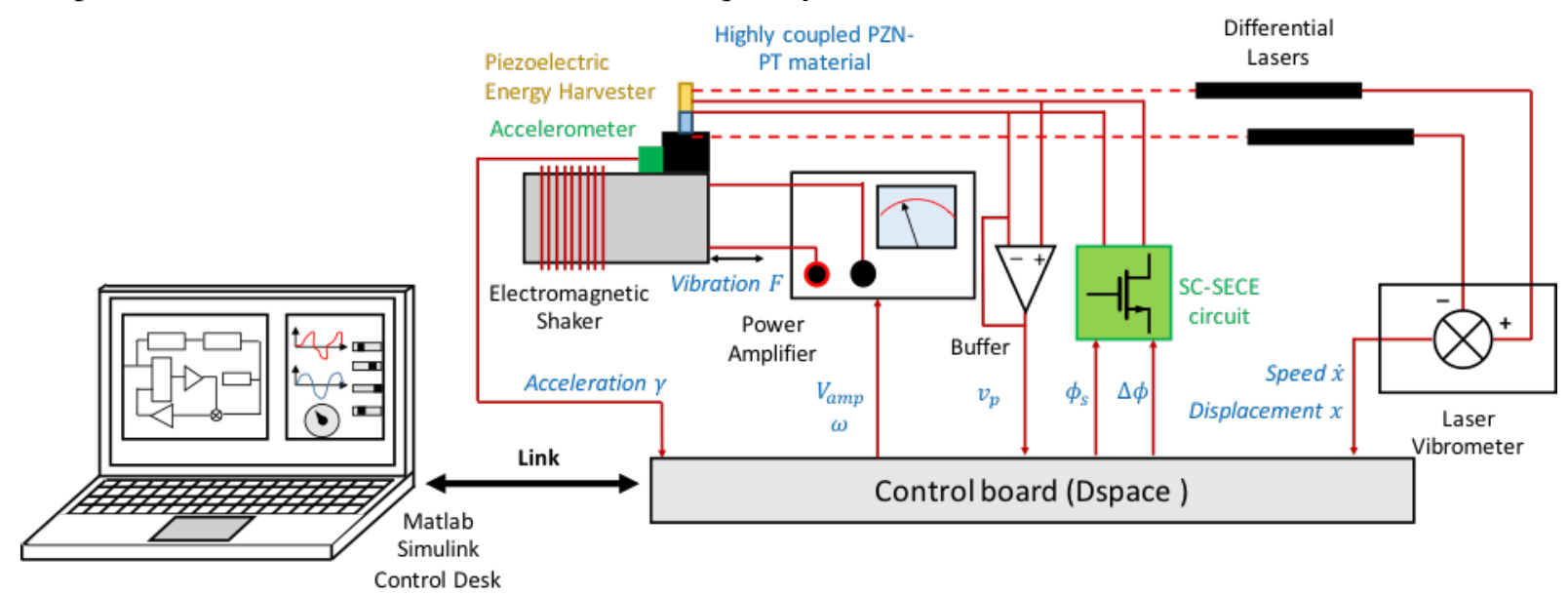

Figure 9 - Experimental setup including a highly coupled PEH

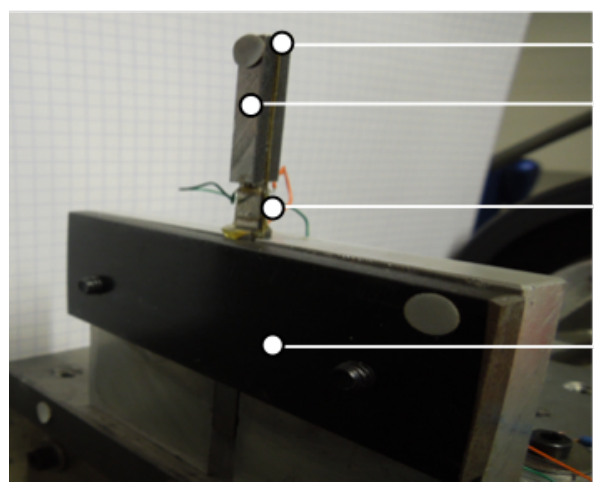

Cantilever beam, $40 \times 5 \times 0.3 \mathrm{~mm}^{3}$

2 Inertial masses in steel, $30 \times 5 \times 2.5 \mathrm{~mm}^{3}$

2 PZN-PT plates, $10 \times 5 \times 0.5 \mathrm{~mm}^{3}$

Figure 10 - Highly coupled piezoelectric harvester

The experimentations are run for 30 values of $\phi_{s}$ between 0 and $\pi, 30$ values of $\Delta \phi$ between 0 and $\pi$, and 100 vibration's frequencies $f_{\text {vib }}$ between $90 \mathrm{~Hz}$ and $140 \mathrm{~Hz}$. The piezoelectric energy harvester's parameters have been summarized in Table 1 .

Table 1. Highly coupled PEH characteristics

\begin{tabular}{ccc}
\hline Parameters names & Parameters values & Units \\
\hline$k^{2}$ & 0.32 & - \\
$k_{m}^{2}$ & 0.48 & - \\
$Q_{m}$ & 20 & - \\
$C_{p}$ & 1.5 & $\mathrm{nF}$
\end{tabular}




\subsection{Experimental results}

At first, the shaker has been excited under a relatively low acceleration amplitude $|\gamma|=$ $0.03 \mathrm{~g}$. Some experimental voltages and displacement waveforms are shown in Fig.11 for various $\left(\phi_{s}, \Delta \phi\right) . v_{\text {control }}$ represents the voltage signal controlling the short-circuit transistor $\left(\mathrm{S}_{2}\right.$ in Fig.3) of the SC-SECE interface. When $v_{\text {control }}$ is set high, the piezoelectric element is short-circuited, and when $v_{\text {control }}$ is set low, the piezoelectric element works in open-circuit configuration. We can observe that the displacement amplitude is sinusoidal, which validates the assumption formulated in (3). Even though the higher mechanical modes were considered negligible in the theoretical part, we can see their impact on the piezoelectric voltage waveforms, on which ripples can be observed. However, these influences remain fairly limited, and does not impact much the harvesting performances of the SC-SECE, as it will be shown further.
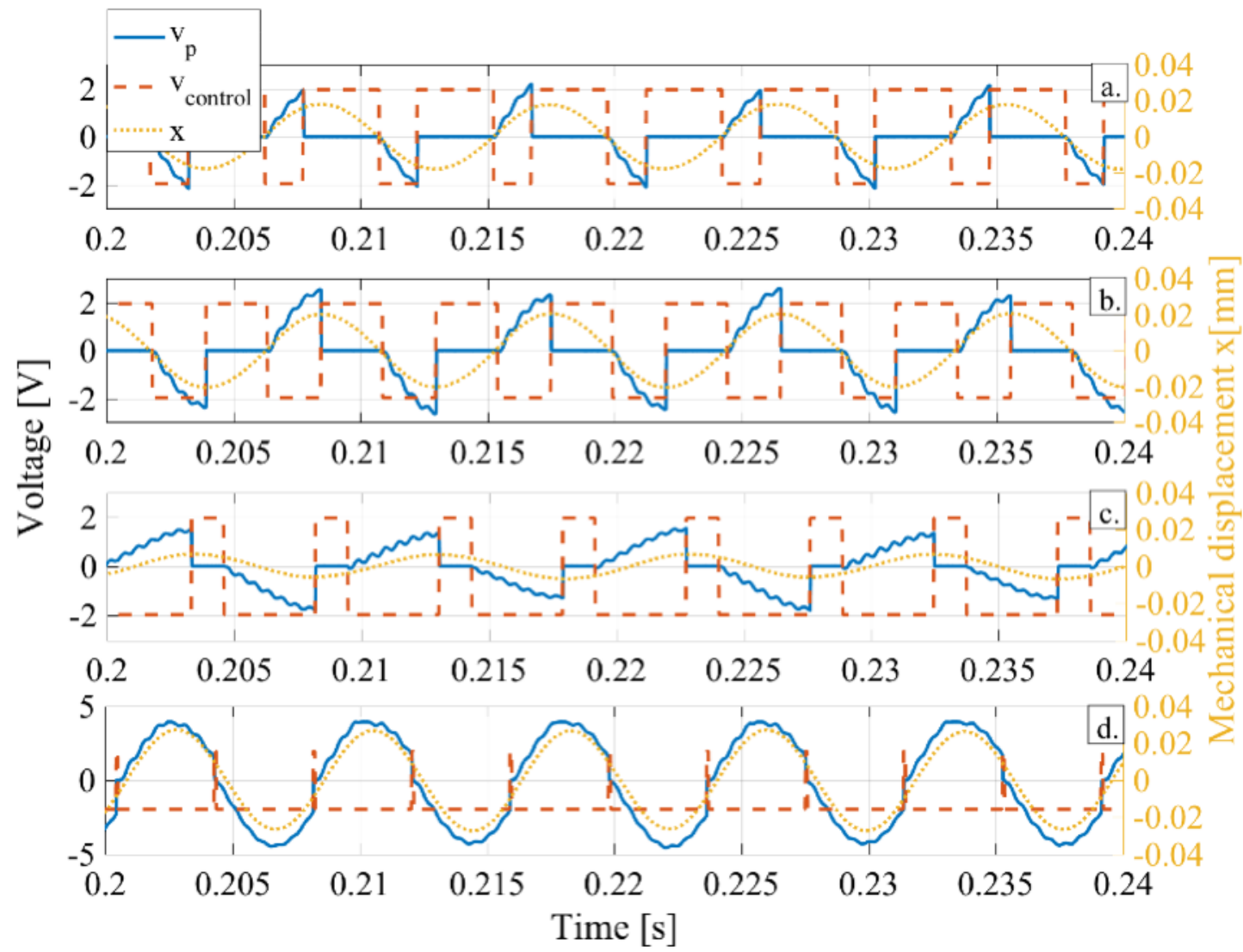

Figure 11 - Experimental waveforms of the proposed SC-SECE with various $\left(\phi_{S}, \Delta \phi\right)$ combinations:

$$
\text { a. }\left(\frac{3 \pi}{4}, \frac{2 \pi}{3}\right) \text { b. }\left(0, \frac{4 \pi}{7}\right) \text { c. }\left(0, \frac{2 \pi}{5}\right) \text { d. }\left(\frac{\pi}{3}, \frac{\pi}{20}\right)
$$

For various vibration frequency, the extracted power from the piezoelectric harvester has been determined experimentally with various $\left(\phi_{s}, \Delta \phi\right)$, as shown in Fig.12. The maximum harvested power with the SC-SECE as well as with the optimal tunable parameters $\left(\phi_{s_{\text {opt }}}, \Delta \phi_{\text {opt }}\right)$ under a small acceleration of $0.03 \mathrm{G}$ is shown in Fig.11. 

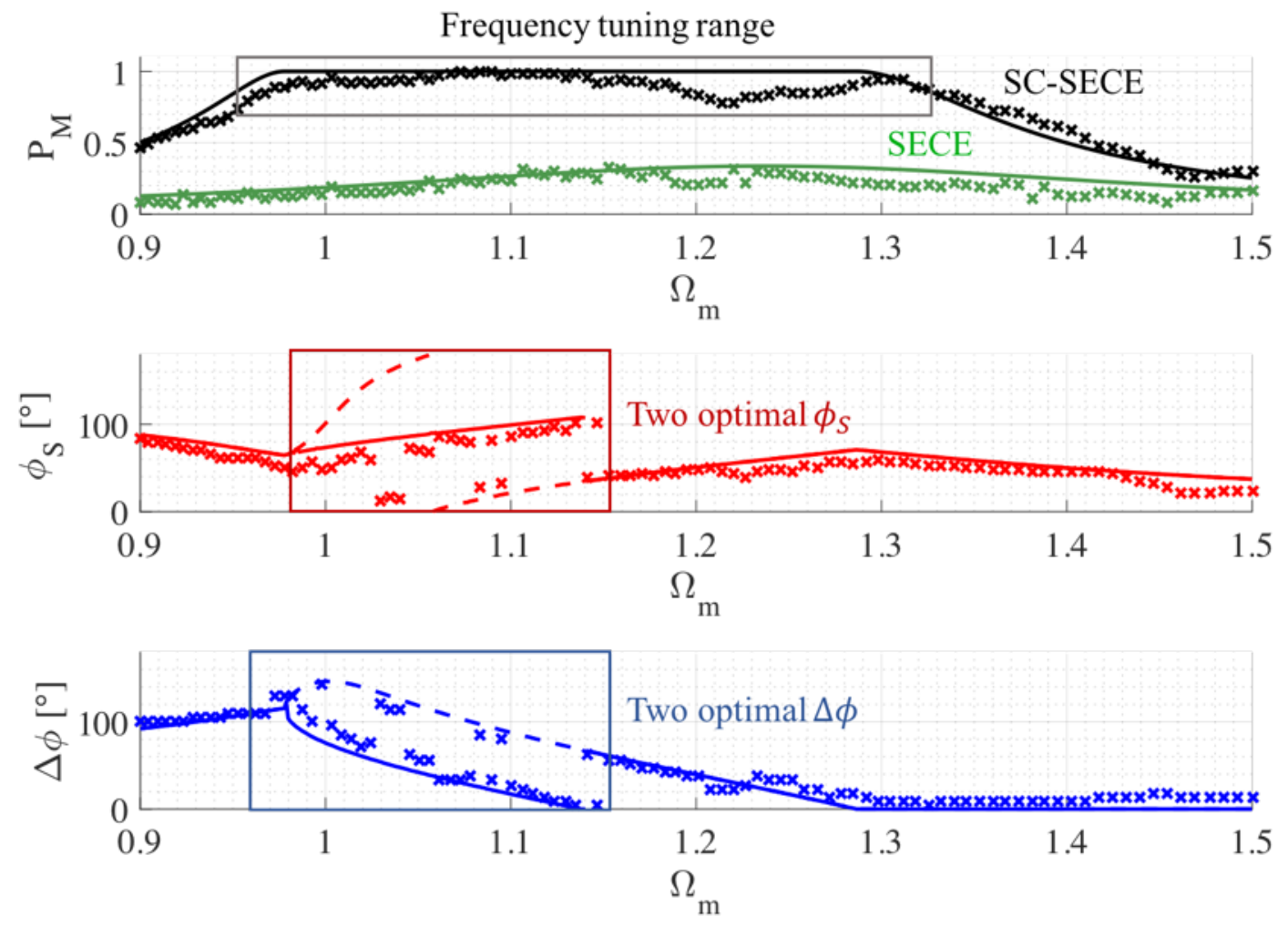

Figure 12 - Experimental (crosses) and theoretical (straight lines) power frequency response of the standard SECE (green) and of the proposed SC-SECE (black) under small vibrations, with $|\gamma|=0.03 G$, and optimal couples $\left(\phi_{s}, \Delta \phi\right)$.

We can see that the experimental results are in good agreement with the harvested power predictions given by (18), even if some noticeable differences can be observed due to higher mechanical modes excitations. This is due to the non-linear treatment of the piezoelectric voltage which stimulates higher harmonics of the mechanical system. Under small vibrations of $0.03 \mathrm{G}$, the maximum harvested power reaches $6 \mu \mathrm{W}$. The proposed SC-SECE strategy allows to harvest more than $3 \mu W$ (which corresponds to $50 \%$ of the maximum harvested power) between $90 \mathrm{~Hz}$ and $140 \mathrm{~Hz}$, which makes the harvesting bandwidth $\Delta \Omega / \Omega_{c}$ as large as $50 \%$ of the short-circuit resonant frequency of the harvester. We can observe a bifurcation of the optimal parameters when $\Omega_{m} \in[0.98,1.15]$. Indeed, on this frequency range, two distinct couples of parameters $\left(\phi_{s}, \Delta \phi\right)$ are leading to two voltage waveforms that share the same (and optimal) first harmonic characteristics (phase and amplitude) even though their spectral contents are different. Experimentally, one of the solutions (straight line) is most of the time a bit more optimal than the other one (dashed line), due to the higher voltage harmonics which have a small impact on the harvested power but have not been considered in the analytical model, due to the first harmonic approximation.

Fig.13.

We ran the same experiment with a higher acceleration amplitude, $|\gamma|=0.08 G$, as shown in 

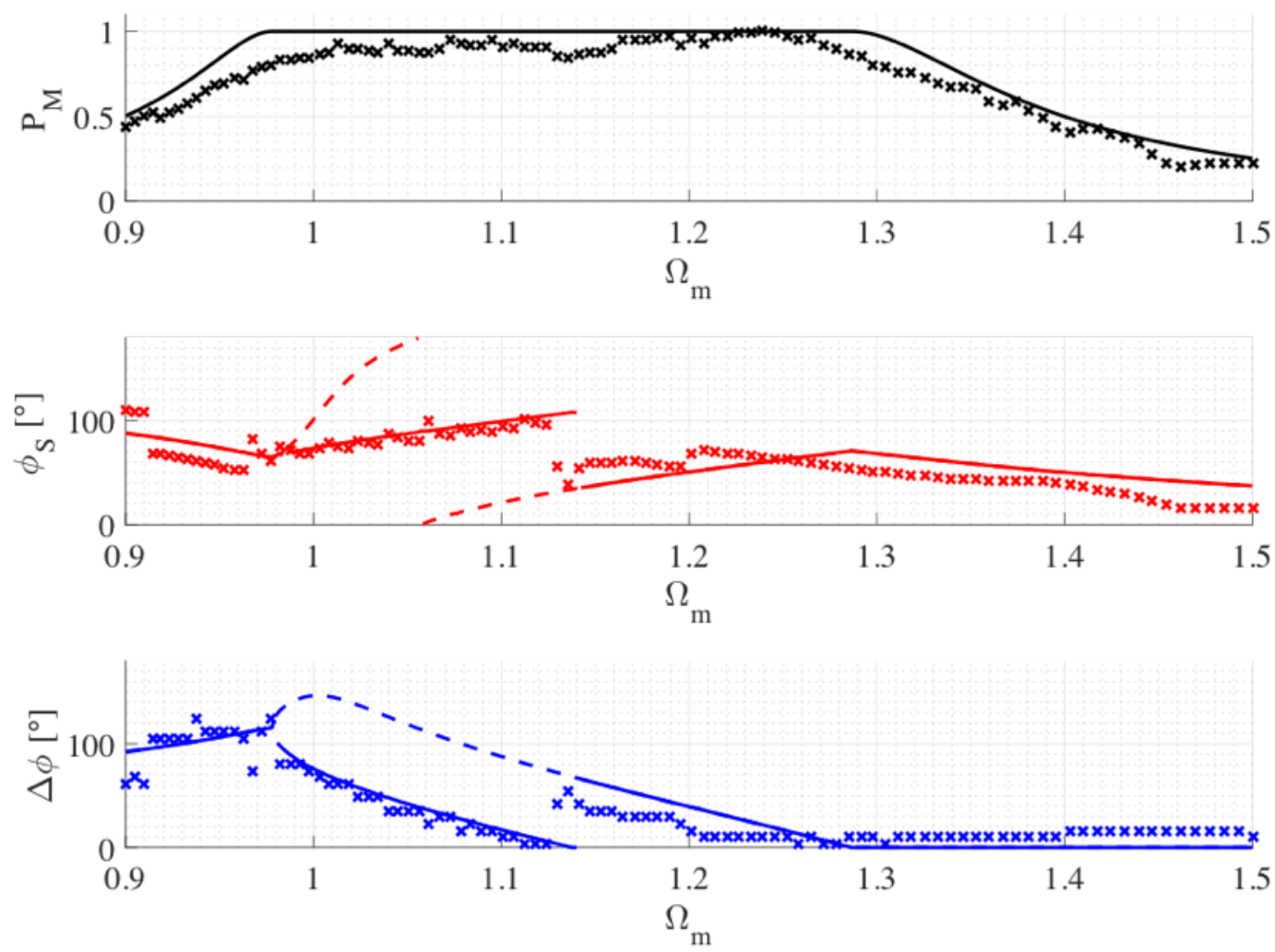

Figure 13 - Experimental (crosses) and theoretical (straight lines) power frequency response of the proposed SC-SECE under small vibrations, with $|\gamma|=0.08 G$, and optimal couples $\left(\phi_{s}, \Delta \phi\right)$.

Overall, the experimental results remain in good agreement with the theoretical model. The maximum harvested power under $0.08 \mathrm{G}$ is $35 \mu \mathrm{W}$, which is a bit lower than what could be predicted by (5). Indeed, under higher acceleration, the quality factor of the mechanical resonator $Q_{m}$ decreases, and is here around 15 . The harvesting bandwidth $\Delta \Omega / \Omega_{c}$ remains around $50 \%$, from $90 \mathrm{~Hz}$ to $140 \mathrm{~Hz}$. On this frequency band, the harvested power is always greater than $15 \mu \mathrm{W}$.

Fig.14.

The same experiment has been finally run with $|\gamma|=0.2 G$. The results are shown below, in 

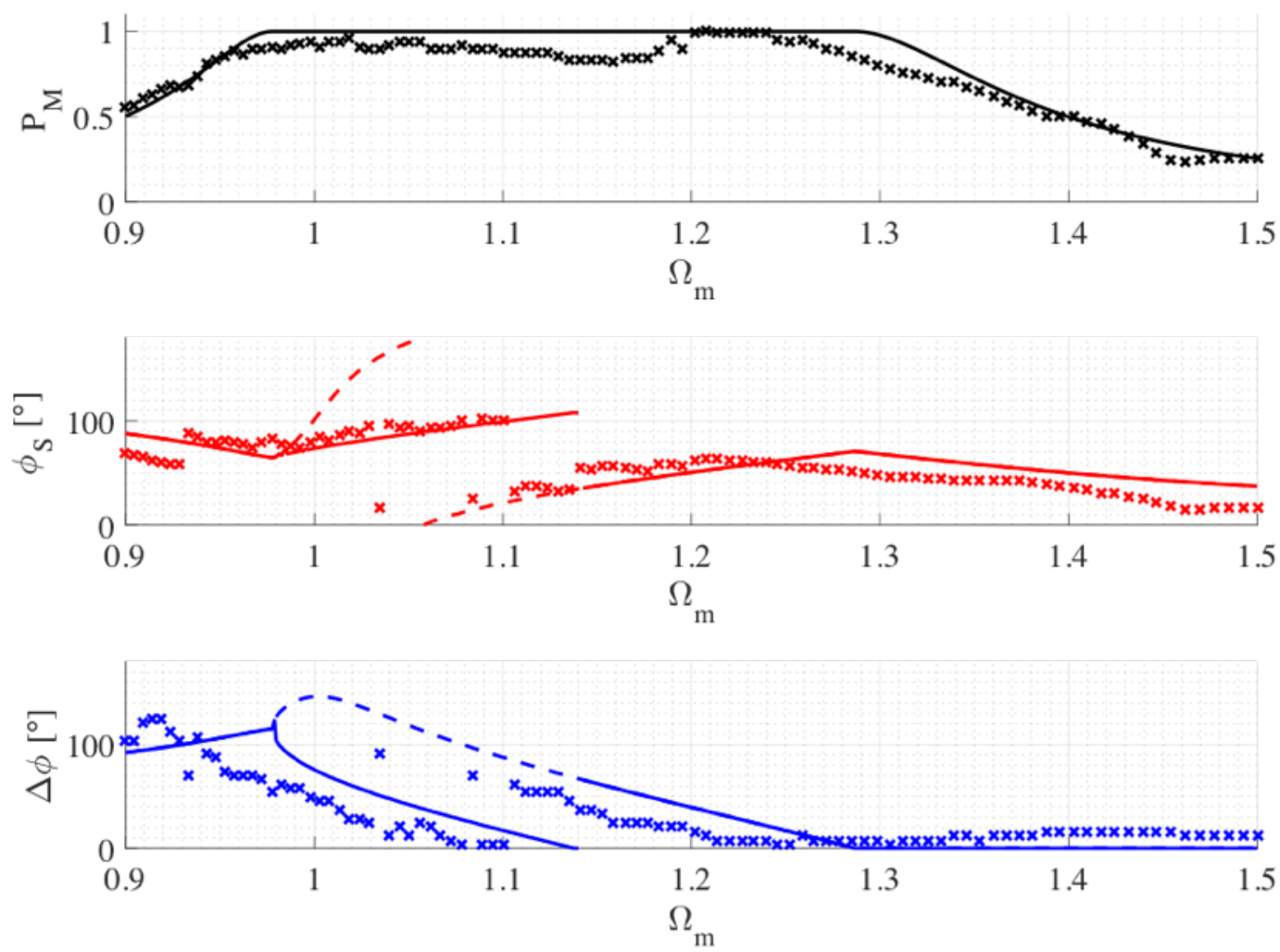

Figure 14 - Experimental (crosses) and theoretical (straight lines) power frequency response of the proposed SC-SECE under small vibrations, with $|\gamma|=0.2 G$, and optimal couples $\left(\phi_{s}, \Delta \phi\right)$.

Under this stronger vibration, the experimental results remain in relatively good agreement with the theoretical predictions, even though we start to observe a shift of the mechanical resonant frequency of the harvester, which seems to have decreased from $100 \mathrm{~Hz}$ to $96 \mathrm{~Hz}$. This is particularly apparent when comparing the experimental and theoretical $\left(\phi_{s}, \Delta \phi\right)$. This shift of the mechanical resonant frequency is due to some mechanical nonlinearities: as the vibration amplitude gets larger, the global stiffness of the beam decreases (softening behavior).

The maximum harvested power under $0.2 G$ is $185 \mu \mathrm{W}$, which is slightly lower than what could be predicted by (5). Once again, the harvesting bandwidth $\Delta \Omega / \Omega_{c}$ is close to $50 \%$, while the harvested power is greater than $92 \mu \mathrm{W}$ between $90 \mathrm{~Hz}$ and $140 \mathrm{~Hz}$. Those experimental results, summarized in Fig. 15, prove that the harvesting bandwidth as well as the power frequency responses of the harvester do not change much with the vibration's amplitude, which confirms the robustness and potential of the SC-SECE approach. 

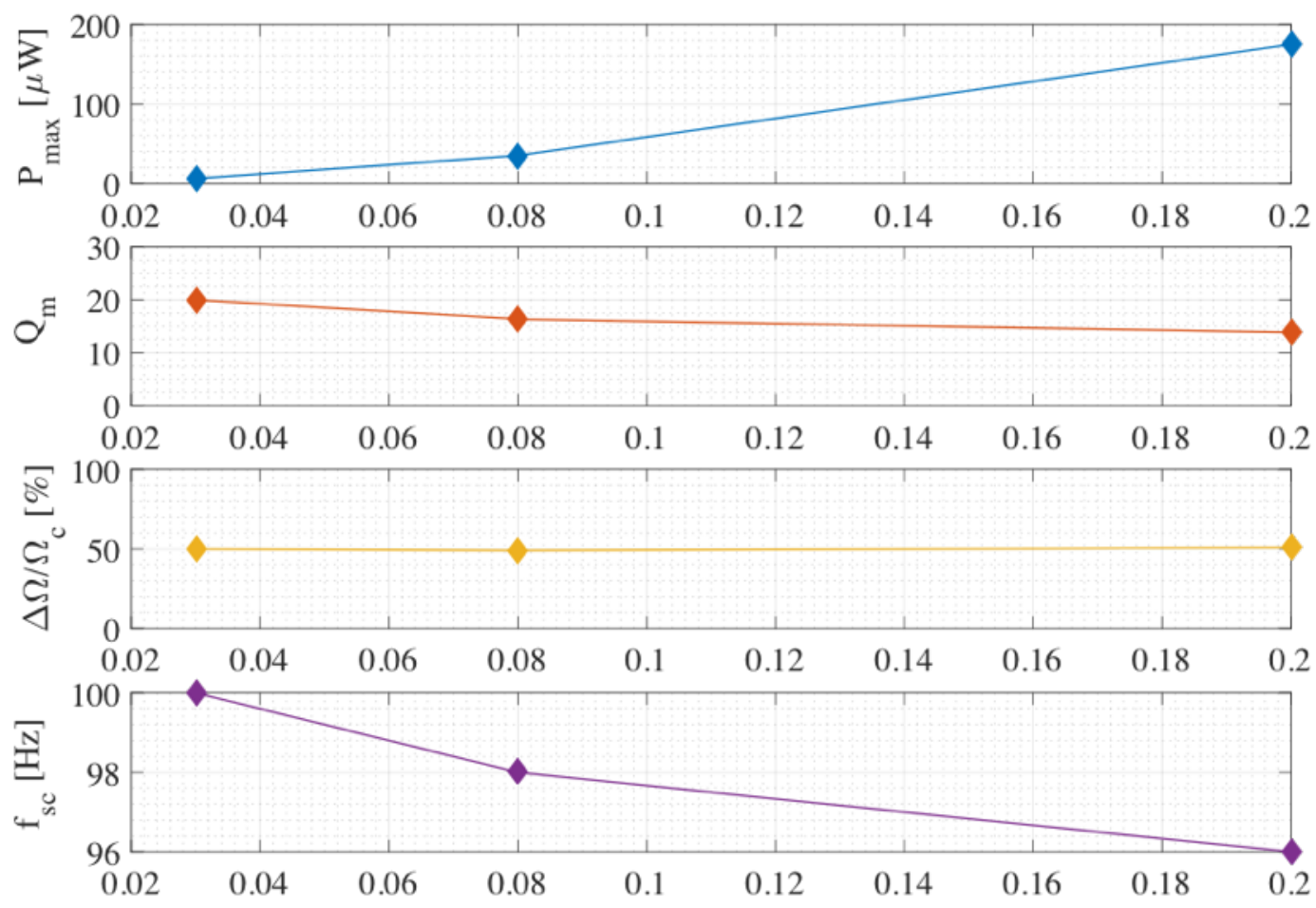

Acceleration amplitude $|\gamma|[\mathrm{G}]$

Figure 15 - Experimental variations of $P_{\max }, Q_{m}, \Delta \Omega / \Omega_{c}$, and $f_{s c}$ with the acceleration amplitude $|\gamma|$

\section{Comparison with other state-of-the-art wideband solutions}

Previous approaches for enlarging the harvesting bandwidth of energy harvesters usually consist in replacing the linear mechanical resonator with a nonlinear one. These nonlinearities can for instance be induced thanks to magnets repulsion and attraction forces, to nonlinear springs, or buckled beam structures. It has been proven in the last decade that such nonlinear harvester can drastically enhance the harvesting bandwidth compared to linear PEHs [10]. Recently, new electrically-based approaches have been investigated such as the FTSECE [17] and the N-SECE [22]. In this section, we compare the experimental results obtained with those different electrical and mechanical approaches with the proposed SC-SECE approach.

\subsection{Figure of merit classifying wideband harvesting solutions}

W. Liu et al. proposed a figure of merit called Systematic Figure of Merit with Bandwidth information $\left(S F o M_{B W}\right)$ in order to classify and categorize wideband piezoelectric harvesters [30]. The expression of this Figure of Merit (FoM) is reminded below:

$$
S F o M_{B W}=E_{H W} \frac{\Delta \Omega}{\Omega_{c}} Q_{m} \rho_{e f f}
$$

Where $E_{H W}=P_{a v} / P_{\max }$ corresponds to the energy harvester effectiveness criterion, $P_{a v}$ being the average harvested power over the considered bandwidth, and $P_{\max }$ being the maximum available power as defined by (5). $\Delta \Omega / \Omega_{c}$ corresponds to the normalized harvesting bandwidth, as 
defined previously. $Q_{m}$ is the quality factor of the harvester and is a good indicator of what the maximum available power $P_{\max }$ is, as formulated in (5). $\rho_{\text {eff }}$ is the effective mass density, defined as $\rho_{\text {eff }}=M / V, M$ being the inertial mass of the harvester, and $V$ the total volume which can be approximated as the sum of the PEH's inertial mass volume $V_{\text {mass }}$ and the motion space $V_{\text {motion }}$. In the case of our highly coupled PEH associated with the SC-SECE interface, $E_{H W}=0.8, \Delta \Omega$ / $\Omega_{c}=0.48, Q_{m}=20, \rho_{\text {eff }}=5700 \mathrm{~kg} \cdot \mathrm{m}^{-3}$, leading to a $S F o M_{B W}$ of about $43776 \mathrm{~kg} \cdot \mathrm{m}^{-3}$. Figure 16 compares the $S F o M_{B W}$ of state-of-the-art wideband solutions with the proposed SC-SECE.

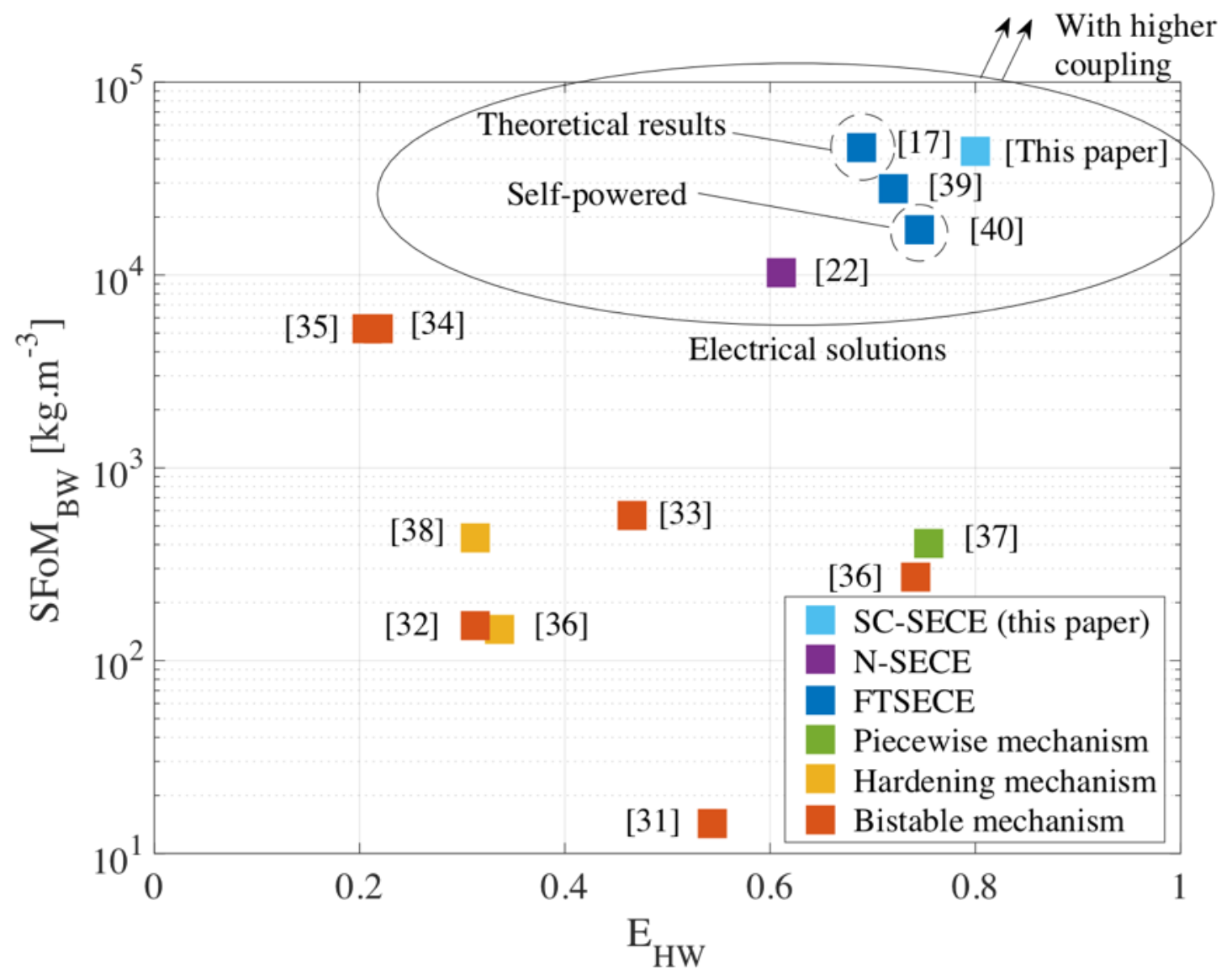

Figure $16-S F o M_{B W}$ of mechanical [31-38] and electrical [17, 22, 39, 40, this paper] solutions

\subsection{Comparison of the SC-SECE with other wideband electrical solutions}

Among the electrical solutions, the most important $S F o M_{B W}$ is exhibited by the FTSECE design proposed in [17]. However, these results are only theoretical, and have not been experimentally validated. The N-SECE proposed in [22] exhibits a lower $S F o M_{B W}$, but only requires a single parameter tuning which is much easier to implement. The FTSECE proposed in [40], while being selfpowered and autonomous, still exhibits a relatively important $S F o M_{B W}$. This design is a proof that an ultra-low-power implementation of such electrical wideband solutions is feasible. Among the electrical solutions which have been experimentally validated, the proposed SC-SECE has the greatest $S F o M_{B W}$, which confirms the potential of the SC-SECE compared to other electrical approaches. 


\subsection{General comparison of electrical solutions with wideband mechanical solutions}

As it can be seen in Fig. 16, electrically-based wideband solutions $S F o M_{B W}$ are substantially higher than the mechanically-based solutions. This is mainly due to two reasons:

- The effective mass densities $\rho$ of the electrical solutions are more important than their mechanical counterparts. Indeed, with equivalent inertial mass, linear harvesters' volumes are usually smaller than nonlinear ones.

- The energy harvester effectiveness criterion $E_{H W}$ is generally greater for electrical solutions.

However, the comparison with wideband mechanical solutions would not be fair without highlighting some limitations of this analysis:

This approach does not consider the power consumption of the control circuit (except for [40]). Indeed, the control circuit as well as the 3-D MPPT loop that aims to control both $\phi_{s}$ and $\Delta \phi$ should consume as few power as possible in order to preserve the efficiency of this approach and not waste all the harvested energy. Nonetheless, we are confident that designing this kind of lowpower interface is possible, since low-power self-powered SECE have already been designed in previous works [40, 41, 42]. 3-D MPPTs have also been implemented in prior art for DC/DC converters, exhibiting energy consumptions as low as $\mu W s$ [43].

- The electrical solutions have been proposed to harvest energy from mono-frequency vibrations, and to track the vibration's frequency with the harvester's resonant one. In the case of multi-frequency harmonic excitations, our circuit, because of its nonlinearity, would not exhibit the frequency response previously shown in the experimental part. Studying the behavior of the SC-SECE under wideband excitations is out of the scope of this paper but may be considered in future researches.

In another hand, we can also underline two main advantages the electrical solutions present and that are not pointed up by the $S F O M_{B W}$ :

- The electrical solutions do not induce any high orbit, nor low orbit behaviors contrary to nonlinear mechanical oscillator for whom several more or less energy-generating behaviors can be observed for the same sinusoidal excitation. If rightly tuned, the electrical solutions will always extract the same amount of energy, which is predictable and hence offer more robustness than the nonlinear solution.

- The electrical solutions bandwidth remains constant with any vibration's amplitude. This is not the case of the nonlinear mechanical solutions, whose frequency responses shapes are dependent on the excitation amplitude.

\section{Conclusion}

In this paper, we proposed a new electrical strategy called SC-SECE. This strategy allows to electrically tune both the resonant frequency and the induced damping of a highly coupled linear piezoelectric harvester. Thanks to the first harmonic analysis, we first proposed an analytical expression of the harvested power, and we determined numerically the power frequency responses of piezoelectric energy harvesters associated with the SC-SECE strategy. Next, we verified experimentally our analytical model on a fabricated highly coupled piezoelectric harvester made with PZN-PT plates. Under very low vibration's amplitude of $0.03 \mathrm{G}$, we have been able to harvest more 
than $4 \mu \mathrm{W}$ on a relatively large frequency band, between $90 \mathrm{~Hz}$ and $140 \mathrm{~Hz}$. Increasing the acceleration up to $0.2 \mathrm{G}$ did not change much the reachable harvesting bandwidth, which remains of approximatively $50 \mathrm{~Hz}$. In a last section we compared the results obtained on this electrically tunable strategy with nonlinear harvesters. The reachable figure of merit seems promising, as long as the consumption of the interface circuit remains considerably smaller than the harvested power. Our future work will focus on the design of a dedicated ultra-low power ASIC realizing this strategy, in order to push forward the proposed concept, and turn it into a self-powered interface circuit.

\section{Acknowledgments}

This work was supported by the French Alternative Energies and Atomic Energy Commission.

\section{References}

[1] M. T. Penella and M. Gasulla, "A review of commercial energy harvesters for autonomous sensors," in Proc. IMTC, pp. 1-5, May 2007.

[2] R. Vullers, R. vanSchaijk, I. Doms, C. vanHoof, and R. Mertens, "Micropower energy harvesting," Solid-State Electron., vol. 53, no. 7, pp. 684-693, 2009.

[3] J. Olivo, S. Carrara, and G. DeMicheli, "Energy harvesting and remote powering for implantable biosensors," IEEE Sensors J., vol. 11, no. 7, pp. 1573-1586, Jul. 2011.

[4] S. Roundy et al., "Improving power output for vibration-based energy scavengers," IEEE Pervasive Computing, vol. 4, no. 1, pp. 28-36, Jan. 2005.

[5] P. Mitcheson, T. Toh, K. Wong, S. Burrow, A. Holmes, "Tuning the Resonant Frequency and Damping of an Electromagnetic Energy Harvester Using Power Electronics," IEEE Trans. Circuits Syst., vol. 58, no. 12, pp. 792-796, December 2011.

[6] T.C. de Godoy, and M. A. Trindade, "Effect of parametric uncertainties on the performances of a piezoelectric energy harvesting device," Journal of the Brazilian Society of Mechanical Sciences and Engineering, vol. 34, no. 2, pp. 552-560, 2012.

[7] S. Boisseau, G. Despesse, and B. A. Seddik, "Nonlinear H-shaped springs to improve efficiency of vibration energy harvesters," Journal of Applied Mechanics, vol. 80, no. 6, 2013.

[8] B. Ando, S. Baglio, A. R. Bulsara, and V. Marletta, "A bistable buckled beam based approach for vibrational energy harvesting," Sens. Actuators A, Phys., vol. 211, pp. 153-161, May 2013.

[9] J-T. Lin, B. Lee, and B. Alphenaar, "The magnetic coupling of a piezoelectric cantilever for enhanced energy harvesting," Smart Mater. Struct., vol. 19, no. 4, pp. 1-7, 2010.

[10] R. L. Harne, and K. W. Wang, "A review of the recent research on vibration harvesting via bistable systems," Smart Mater. Struct., vol. 22, no. 2, pp. 1-7, 2010.

[11] M. F. Daqaq, R. Masana, A. Erturk, and D. D. Quinn, "On the role of nonlinearities in vibratory energy harvesting: A critical review and discussion," Appl. Mech. Rev., vol. 66, no. 4, p. 040801, 2014. 
[12] A. Badel, E. Lefeuvre, "Nonlinear Conditioning Circuits for Piezoelectric Energy Harvesters", in: E. Blokhina, A.E. Aroudi, E. Alarcon, D. Galayko (Eds.), Nonlinearity Energy Harvest. Syst., Springer International Publishing, pp. 321-359, 2016.

[13] J. Liang and W. H. Liao, "Impedance Modeling and Analysis for Piezoelectric Energy Harvesting Systems," IEEE/ASME Trans. Mechatron., vol. 17, no. 6, pp. 1145-1157, Dec. 2012.

[14] W.-J. Wu, Y.-Y. Chen, B.-S. Lee, J.-J. He, and Y.-T. Peng, "Tunable resonant frequency power harvesting devices," in Smart Structures and Materials 2006: Damping and Isolation, vol. 6169, pp. 61690A, 2006.

[15] Y. Liao and H. A. Sodano, "Optimal parameters and power characteristics of piezoelectric energy harvesters with an RC circuit," Smart Mater. Struct., vol. 18, no. 4, p. 045011, Mar. 2009.

[16] B. Ahmed-Seddik, G. Despesse, S. Boisseau, E. Defay, "Self-powered resonant frequency tuning for piezoelectric vibration energy harvesters," Journal of Physics: Conference Series, vol. 476, pp. $012069,2013$.

[17] A. Badel and E. Lefeuvre, "Wideband Piezoelectric Energy Harvester Tuned Through its Electronic Interface Circuit," Journal of Physics: Conference Series, vol. 557, pp. 12115, 2014.

[18] Y. Cai, Y. Manoli, "System design of a time-controlled broadband piezoelectric energy harvesting interface circuit," in proc. IEEE International Symposium on Circuits and Systems, pp. 237-240, 2015.

[19] P. H. Hsieh, C.-H. Chen, and H.-C. Chen, "Improving the scavenged power of nonlinear piezoelectric energy harvesting interface at off-resonance by introducing switching delay," IEEE Trans. Power Electron., vol. 30, pp. 3142-3155, June 2015.

[20] B. Zhao, J. Liang, and K. Zhao, "Phase-variable Control of Parallel Synchronized Triple BiasFlips Interface Circuit towards Broadband Piezoelectric Energy Harvesting," in 2018 IEEE International Symposium on Circuits and Systems (ISCAS), pp. 1-5, 2018.

[21] J. Liang, "Synchronized bias-flip interface circuits for piezoelectric energy harvesting enhancement: A general model and prospects," J. Intell. Mater. Syst. Struct., vol. 28, no. 3, pp. 339$356,2017$.

[22] A. Morel, A. Badel, Y. Wanderoild, G. Pillonnet, "A unified N-SECE strategy for highly coupled piezoelectric energy scavengers," Smart Mater. Struct., in press.

[23] A. Morel, P. Gasnier, Y. Wanderoild, G. Pillonnet, A. Badel, "Short Circuit Synchronous Electric Charge Extraction (SC-SECE) Strategy for Wideband Vibration Energy Harvesting," in proc. IEEE International Symposium on Circuits and Systems, pp. 1-5, 2018.

[24] X Wang (2016). Frequency Analysis of Vibration Energy Harvesting Systems, Elsevier, London, UK, ISBN: 9780128023211

[25] E. Arroyo, A. Badel, F. Formosa, Y. P. Wu, and J. Qiu, "Comparison of electromagnetic and piezoelectric vibration energy harvesters: Model and experiments," Sensors and Actuators A: Physical, vol. 183, pp. 148-156, 2012. 
[26] E. Lefeuvre, A. Badel, C. Richard, and D. Guyomar, "Piezoelectric Energy Harvesting Device Optimization by Synchronous Electric Charge Extraction," J. Intell. Mater. Syst. Struct., vol. 16, no. 10, pp. 865-876, Oct. 2005.

[27] D. Guyomar and M. Lallart, "Recent progress in piezoelectric conversion and energy harvesting using nonlinear electronic interfaces and issues in small scale implementation," Micromachines, vol. 2, pp. 274-294, 2011.

[28] X. Wang and L. W. Lin, "Dimensionless Optimization of Piezoelectric Vibration Energy Harvesters with Different Interface Circuits," Smart Mater. Struct, Vol. 22, pp.1-20, ISSN: 0964-1726, 2013.

[29] A. Morel, G. Pillonnet, A. Badel, "Regenerative synchronous electrical charge extraction for highly coupled piezoelectric generators," in proc. International Midwest Symposium on Circuits and Systems, pp. 237-240, 2011.

[30] W. Q. Liu, A. Badel, F. Formosa, Y. P. Liu, "A new figure of merit for wideband vibration energy harvesters,” Smart Mater. Struct., vol. 24, no. 12, 2015.

[31] S. C. Stanton, C. C. Mcgehee, and B. P. Mann, "Nonlinear dynamics for broadband energy harvesting: investigation of a bistable piezoelectric inertial generator" Physica D, vol. 239, no. 10, pp. 640-53, 2010.

[32] A. Arrieta, and P. Hagedorn "A piezoelectric bistable plate for nonlinear broadband energy harvesting” Appl. Phys. Lett., vol. 97, no. 10, 2010.

[33] B. P. Mann, and B. A. Owens, "Investigations of a nonlinear energy harvester with a bistable potential well," J. Sound Vib., vol. 329, pp. 1215-26, 2010.

[34] W. Q. Liu, A. Badel, F. Formosa, Y. Wu and A. Agbossou, "Novel piezoelectric bistable oscillator architecture for wideband vibration energy harvesting," Smart Mater. Struct., vol. 22, no. 3 , 2013.

[35] W. Q. Liu, A. Badel, F. Formosa, Y. Wu, N. Bencheikh, and A. Agbossou, "A wideband integrated piezoelectric bistable generator: experimental performance evaluation and potential for real environmental vibrations,” J. Intell. Mater. Syst. Struct., vol. 26, no. 7, pp. 872-877, 2015.

[36] R. Masana, and M. F. Daqaq, "Relative performance of a vibratory energy harvester in mono- and bistable potentials," J. Sound Vib., vol. 330, no. 24, pp. 6036-52, 2011.

[37] K. Ashraf, M. H. K. Khir, J. O. Dennis, and Z. Baharudin, "A wideband, frequency up-converting bounded vibration energy harvester for a low-frequency environment," Smart Mater. Struct., vol. 22, no. 2, 2013.

[38] G. Sebald, H. Kuwano, D. Guyomar, and B. Ducharne, "Simulation of a Duffing oscillator for broadband piezoelectric energy harvesting” Smart Mater. Struct., vol. 20, no. 7, 2011.

[39] A. Brenes, E. Lefeuvre and C.-S. Yoo, "Experimental validation of wideband piezoelectric energy harvesting based on frequency-tuning synchronized charge extraction," Journal of Physics: Conference Series, vol. 1052, pp. 012050, 2018. 
[40] Y. Cai and Y. Manoli, "A Piezoelectric Energy-Harvesting Interface Circuit with Fully Autonomous Conjugate Impedance Matching, 156\% Extended Bandwidth, and $0.38 \mu \mathrm{W}$ Power Consumption," in proc. IEEE International Solid State Circuit Conference, pp. 148-150, 2018.

[41] T. Hehn, F. Hagedorn, D. Maurath, D. Marinkovic, I. Kuehne, A. Frey, and Y. Manoli, "A fully autonomous integrated interface circuit for piezoelectric harvesters," Journal of Solid-State Circuits, vol. 47, pp. 2185-2198, September 2012.

[42] A. Morel, A. Quelen, P. Gasnier, R. Grézaud, S. Monfray, A. Badel, and G. Pillonnet, "A ShockOptimized SECE Integrated Circuit," Journal of Solid-State Circuits, in press.

[43] K. Rawy, T. Yoo, T. T.-H. Kim, “An 88\% Efficiency 0.1-300- $\mu$ W Energy Harvesting System with 3-D MPPT Using Switch Modulation for IoT Smart Nodes," Journal of Solid-State Circuits, vol. PP, no. 99, 2018. 